\title{
Normalization of type IIB D-instanton amplitudes
}

\section{Ashoke Sen}

Harish-Chandra Research Institute, HBNI,

Chhatnag Road, Jhusi, Allahabad 211019, India

E-mail: sen@hri.res.in

ABSTRACT: We compute the normalization of single D-instanton amplitudes in type IIB string theory and show that the result agrees with the prediction of S-duality due to Green and Gutperle.

Keywords: D-branes, String Field Theory

ARXIV EPrint: 2104.11109 


\section{Contents}

1 Introduction and summary 1

2 Conventions for the world-sheet theory 4

3 Conventions for string field theory $\quad 8$

$\begin{array}{llr}3.1 & \text { Closed string sector of string field theory } & 8\end{array}$

$\begin{array}{lll}3.2 & \text { Open string sector of string field theory } & 11\end{array}$

$\begin{array}{lll}3.3 & \text { Interaction between open and closed strings } & 13\end{array}$

$\begin{array}{ll}3.4 & \text { Relation between the different coupling constants } \\ \end{array}$

4 Normalization of the D-instanton amplitude $\quad 15$

$\begin{array}{ll}4.1 \text { Annulus partition function } & 16\end{array}$

$\begin{array}{ll}\text { 4.2 Gauge invariant string field theory in the } L_{0}=0 \text { sector } & 18\end{array}$

$\begin{array}{lll}4.3 & \text { Gauge fixing to Siegel gauge } & 19\end{array}$

$\begin{array}{ll}4.4 \text { Gauge invariant partition function } & 20\end{array}$

5 The multiplier factor $\quad 23$

$6 \quad$ 4-graviton amplitude $\quad 24$

7 Prediction for the D-instanton contribution to the four graviton ampli$\begin{array}{ll}\text { tude from duality } & 29\end{array}$

$\begin{array}{lll}8 & \text { Generalizations } & 29\end{array}$

A Sphere four point function from sewing of two three point functions $\quad 31$

B Comparison of the two definitions of the brane tension 32

\section{Introduction and summary}

It has been known for many years that string theory amplitudes receive non-perturbative contribution from D-instantons [1,2]. Many D-instanton induced terms were predicted using S-duality invariances of various compactified string theories [2-25], but except for the early attempts [2], the direct systematic computation of these amplitudes from first principles has not been carried out. However during the last two years progress was made in the context of two dimensional string theory [26-31]. In particular, [26, 27] computed the precise contributions to the amplitudes from D-instantons in terms of some constants that appear to be divergent in the world-sheet formalism. It was then found that string field theory gives finite, unambiguous values of these constants [28-31]. 
The goal of this paper will be to extend this analysis to type IIB string theory and verify one of the predictions of S-duality. Our focus will be on the simplest case of ten dimensional type IIB string theory. Tree level four graviton amplitude in this theory receives a correction proportional to $\zeta(3)$ from an eight derivative term in the effective action [32]. This contribution is not invariant under S-duality but can be made S-duality invariant by adding one loop and non-perturbative corrections to the amplitude $[2,3]$. The result takes the form: ${ }^{1}$

$\frac{i}{4} 2^{6} \pi^{7} g_{s}^{2} K_{c}\left[2 \zeta(3)+\frac{2 \pi^{2}}{3} g_{s}^{2}+4 \pi g_{s}^{3 / 2}\left\{e^{2 \pi i \tau}+e^{-2 \pi i \bar{\tau}}\right\}+\cdots\right](2 \pi)^{10} \delta^{(10)}\left(k_{1}+k_{2}+k_{3}+k_{4}\right)$,

where $\tau=a+i g_{s}^{-1}, g_{s}$ is the string coupling defined so that the D-instanton action is given by $2 \pi / g_{s}, a$ is the vacuum expectation value of the RR scalar field and $K_{c}$ is a kinematic factor depending on the momenta $\left\{k_{i}\right\}$ and polarizations $\left\{e^{(i)}\right\}$ of the external graviton states, as described in (6.25), (6.26). The expression (1.1) has been written in the string frame, as should be clear from the explicit factor of $g_{s}^{2}$ multiplying the tree level term proportional to $\zeta(3)$. In the Einstein frame the expression (1.1) is multiplied by a factor of $g_{s}^{-7 / 2}$ and becomes proportional to the S-duality invariant function $E_{3 / 2}(\tau, \bar{\tau})[2,3]$. The one loop term $2 \pi^{2} g_{s}^{2} / 3$ in (1.1) is known to agree with the results of explicit computation [35, 36]. In this paper we shall verify that the leading non-perturbative term proportional to $e^{2 \pi i \tau}$ also agrees with the leading D-instanton contribution to this amplitude.

Formally the leading D-instanton contribution to the four graviton amplitude is given by the product of four disk amplitudes, each with a single graviton vertex operator and four open string fermion zero mode insertions [2]. This part of the amplitude can be computed using straightforward world-sheet methods. However the amplitude is multiplied by an overall normalization factor that can be formally identified as the exponential of the annulus amplitude with no vertex operator insertion. Physically it represents the one loop determinant of the open string fields on the D-instanton. Due to cancellation between the contributions from the NS and R sector states the annulus partition function vanishes and if we take this literally, it would appear that the normalization factor is unity. However, this is deceptive since the contribution from the zero modes cannot be represented as a determinant and the zero mode integrations must be carried out separately. To deal with this we proceed as follows:

1. First we show that the exponential of the annulus partition function can be formally expressed as an integral over the bosonic and fermionic modes of the open string with precise normalization. Since there is no subtlety in the non-zero mode sector, the vanishing of the annulus partition function implies cancellation between the integrals over the non-zero modes of the open string and we focus on the zero mode sector integrals.

2. Then we show that the integral over the zero modes can be regarded as the result of Siegel gauge fixing of a gauge invariant integral over the (zero dimensional)

\footnotetext{
${ }^{1}$ The prediction of S-duality was shown to be consistent with some results in $\mathcal{N}=4$ super Yang-Mills theory via AdS/CFT correspondence [33]. This is also consistent with the analysis of graviton scattering amplitude using S-matrix bootstrap [34].
} 
open string fields. The gauge fixing is done following the standard Faddeev-Popov formalism.

3. Some of the zero modes in the gauge fixed version represent bosonic and fermionic collective modes and must be treated carefully. However one pair of fermionic zero modes in the NS sector can be identified as the Faddeev-Popov ghosts arising from gauge fixing. The vanishing of the quadratic term of the action of these modes indicate the vanishing of the Faddeev-Popov determinant and hence the breakdown of the Siegel gauge [37, 38].

4. We avoid this problem with gauge fixing by using the original gauge invariant version of the path integral instead of the Siegel gauge fixed version. Since the normalization of the gauge fixed version was known, this fixes the normalization of the gauge invariant version. This version does not have integration over the Faddeev-Popov ghost modes, but has an extra integral over an out of Siegel gauge mode of the open string. It also has division by the volume of the gauge group.

5. The out of Siegel gauge mode gives a non-zero contribution to the action. The integration over this mode takes the form of a Gaussian integral and can be carried out explicitly.

6. We find the volume of the gauge group by relating the string field theory gauge transformation parameter $\theta$ to the rigid $\mathrm{U}(1)$ gauge transformation parameter $\widetilde{\theta}$ under which an open string connecting the original D-instanton to a spectator D-instanton picks up a phase $e^{\widetilde{i \theta}}$. This relationship is found by comparing the gauge transformation laws in string field theory to the rigid $\mathrm{U}(1)$ gauge transformation laws. Once this is done we can express the integration over $\theta$ in terms of integration over $\widetilde{\theta}$ and then use the fact that $\widetilde{\theta}$ has period $2 \pi$ to compute the volume of the gauge group.

7. The remaining modes in the NS sector represent bosonic zero modes related to collective modes of the D-instanton describing its location in space-time. We determine the precise normalization relating the two sets of modes by comparing the coupling of the open string zero modes to closed strings to the expected coupling of the collective modes to closed strings. Using this we can express the integration over these bosonic zero modes in terms of integration over the collective modes with some specific normalization factor. The integration over the collective modes is left aside, to be done at the end after combining the contribution from all the pieces. The final integration over these modes generate the usual energy-momentum conserving delta function $(2 \pi)^{10} \delta^{(10)}\left(\sum_{i} p_{i}\right)$.

8. In the $\mathrm{R}$ sector there are 16 fermion zero modes, and all of these can be related to the fermionic collective modes of the D-instanton associated with broken supersymmetry. Integration over these modes is also set aside till the end after we combine all the pieces. In particular, we need to insert 16 fermionic modes into the four disks, each carrying a single graviton vertex operator. The integration over the fermionic collective modes now produces a suitable 16-dimensional $\epsilon$ tensor that needs to be combined with the rest of the amplitude. 
The answer for the one instanton contribution to the four graviton amplitude, computed this way, takes the form:

$$
i e^{2 \pi i a} e^{-2 \pi / g_{s}} 2^{6} \pi^{8} g_{s}^{7 / 2} K_{c}(2 \pi)^{10} \delta^{(10)}\left(k_{1}+k_{2}+k_{3}+k_{4}\right) .
$$

EQ. (1.2) agrees with the term proportional to $e^{2 \pi i \tau}$ in (1.1). $a$ dependence of the amplitude can be obtained by exponentiating the disk one point function of the RR scalar field since that is the only amplitude that involves $a$ and not its derivative. The overall phase of the term is not determined due to the usual ambiguities in evaluating path integral over chiral fermions, but this phase can be absorbed into a shift of $a$.

The fact that the instanton contribution gives the correct dependence on $g_{s}$ was already noted in [39]. The ratio of the subleading non-perturbative corrections, hidden in the $\cdots$ in (1.1), to the leading non-perturbative correction is also being analyzed in [39].

The rest of the paper is organized as follows. In sction 2 we describe our normalization conventions in the world-sheet string theory and compare them with those of [40] whose results we use. In sction 3 we describe our normalization conventions in string field theory, and compare the coupling constants and fields that arise there with those appearing in [40]. Sections 4-6 contain the main results of this paper. In sction 4 we compute the normalization of the D-instanton amplitudes by manipulating the exponential of the annulus zero point function following the procedure described earlier in this section. This computes the total contribution from the steepest descent contour passing through the instanton. However the actual contribution of the instanton to the full amplitude depends on how the steepest descent contour fits inside the actual integration contour. This produces a multiplier factor that accompanies the normalization. In sction 5 we argue that for the D-instanton of type IIB string theory this multiplier factor is one. In sction 6 we compute the disk amplitude with one graviton and four fermionic open string zero mode insertions and combine this with the result of sction 4 to compute the leading D-instanton contribution to the four graviton amplitude. In sction 7 we review the prediction of S-duality for this amplitude and show that the result of explicit D-instanton calculation agrees with the prediction of S-duality. In sction 8 we discuss possible generalization of this analysis to D-instanton contribution in other (compactified) string theories, including the contribution from Euclidean D-branes wrapped along compact cycles.

\section{Conventions for the world-sheet theory}

In this section we shall describe our normalization conventions. Since we are trying to reproduce a single constant, it is important that we carefully keep track of all the constants in our analysis. We work in the $\alpha^{\prime}=1$ unit. For the rest of the conventions, we shall try to follow closely the ones used in [41]. In a few places we shall differ from the convention of [41]; we shall mention them as we encounter these differences.

The world-sheet of type IIB string theory has a set of 10 scalar fields $X^{\mu}$ describing the target space-time coordinates, their superpartner left and right-moving fermions $\psi^{\mu}, \bar{\psi}^{\mu}$, the world-sheet grassmann odd ghost fields $b, c, \bar{b}, \bar{c}$ and the grassmann even ghost fields 
$\beta, \gamma, \bar{\beta}, \bar{\gamma}$. The $\beta, \gamma$ system is 'bosonized' by introducing scalar fields $\phi, \bar{\phi}$, and fermionic fields $\xi, \eta, \bar{\xi}, \bar{\eta}$ via the relations:

$$
\beta=\partial \xi e^{-\phi}, \quad \gamma=\eta e^{\phi}, \quad \bar{\beta}=\bar{\partial} \bar{\xi} e^{-\bar{\phi}}, \quad \bar{\gamma}=\bar{\eta} e^{\bar{\phi}} .
$$

The operator products between various fields take the form:

$$
\begin{aligned}
\partial X^{\mu}(z) \partial X^{\nu}(w) & =-\frac{\eta^{\mu \nu}}{2(z-w)^{2}}+\cdots, \quad \psi^{\mu}(z) \psi^{\nu}(w)=-\frac{\eta^{\mu \nu}}{2(z-w)}+\cdots, \\
c(z) b(w) & =(z-w)^{-1}+\cdots, \\
\xi(z) \eta(w) & =(z-w)^{-1}+\cdots, \\
e^{q_{1} \phi(z)} e^{q_{2} \phi(w)} & =(z-w)^{-q_{1} q_{2}} e^{\left(q_{1}+q_{2}\right) \phi(w)}+\cdots, \\
\partial \phi(z) \partial \phi(w) & =-\frac{1}{(z-w)^{2}}+\cdots,
\end{aligned}
$$

where $\cdots$ denote less singular terms whose knowledge will not be needed for our analysis. The Minkowski metric $\eta^{\mu \nu}$ is taken to have mostly + signature, and is replaced by $\delta_{\mu \nu}$ in the euclidean computation. There are similar operator product expansions involving anti-holomorphic fields that we have not written down. In the following discussion we shall only write down the various relations involving the holomorphic fields, with the implicit understanding that there are similar relations involving anti-holomorphic fields as well.

We assign ghost number 1 to $c, \bar{c}, \gamma, \bar{\gamma}, \eta, \bar{\eta},-1$ to $b, \bar{b}, \beta, \bar{\beta}, \xi, \bar{\xi}$ and 0 to the rest of the fields. We also assign picture number $q$ to $e^{q \phi}$ and $e^{q \bar{\phi}}, 1$ to $\xi, \bar{\xi},-1$ to $\eta, \bar{\eta}$ and 0 to the rest of the fields. The $\mathrm{SL}(2, \mathrm{C})$ invariant vacuum carries zero ghost number and picture number.

The stress tensor $T(z)$ and its fermionic partner $T_{F}(z)$ for the matter sector take the form:

$$
T_{m}(z)=-\partial X^{\mu} \partial X^{\nu} \eta_{\mu \nu}+\psi_{\mu} \partial \psi^{\mu}, \quad T_{F}(z)=-\psi_{\mu} \partial X^{\mu},
$$

with similar expressions for their anti-holomorphic counterparts. The operator product expansions involving $T_{m}$ and $T_{F}$ take the form:

$$
\begin{aligned}
& T_{m}(z) T_{m}(w)=\frac{15}{2} \frac{1}{(z-w)^{4}}+\frac{2}{(z-w)^{2}} T_{m}(w)+\frac{1}{z-w} \partial T_{m}(w)+\cdots \\
& T_{F}(z) T_{F}(w)=\frac{5}{2} \frac{1}{(z-w)^{3}}+\frac{1}{2} \frac{1}{z-w} T_{m}(w)+\cdots \\
& T_{m}(z) T_{F}(w)=\frac{3}{2} \frac{1}{(z-w)^{2}} T_{F}(w)+\frac{1}{z-w} \partial T_{F}(w)+\cdots
\end{aligned}
$$

The stress tensors of the ghost fields are given by

$$
T_{b, c}=-2 b \partial c+c \partial b, \quad T_{\beta, \gamma}(z)=\frac{3}{2} \beta \partial \gamma+\frac{1}{2} \gamma \partial \beta=T_{\phi}+T_{\eta, \xi}
$$

where

$$
T_{\eta, \xi}=-\eta \partial \xi, \quad T_{\phi}=-\frac{1}{2} \partial \phi \partial \phi-\partial^{2} \phi
$$


The BRST charge is given by

$$
Q_{B}=\oint d z \jmath_{B}(z)
$$

where

$$
\jmath_{B}(z)=c(z)\left\{T_{m}(z)+T_{\beta, \gamma}(z)\right\}+\gamma(z) T_{F}(z)+b(z) c(z) \partial c(z)-\frac{1}{4} \gamma(z)^{2} b(z),
$$

and $\oint$ is normalized to include the $1 /(2 \pi i)$ factor so that $\oint d z / z=1$.

The picture changing operator $(\mathrm{PCO}) \mathcal{X}[42,43]$ will be taken to be:

$$
\mathcal{X}(z)=2\left\{Q_{B}, \xi(z)\right\}=2 c \partial \xi+2 e^{\phi} T_{F}-\frac{1}{2} \partial \eta e^{2 \phi} b-\frac{1}{2} \partial\left(\eta e^{2 \phi} b\right) .
$$

This differs from the one used in [41] by a factor of 2 . Since the picture number nonconservation on a Riemann surface of genus $g$ is proportional to $2 g-2$, and since string amplitudes carry factors of $g_{s}^{2 g-2}$ where $g_{s}$ is the string coupling constant, the difference in the normalization of the PCO can be absorbed into a redefinition of the string coupling and the normalization of the vertex operators. We shall see that (2.9) is a convenient normalization to use for computation of amplitudes.

We also introduce the inverse picture changing operator

$$
\mathcal{Y}=2 c \partial \xi e^{-2 \phi} .
$$

Both $\mathcal{X}$ and $\mathcal{Y}$ commute with the BRST operator. Furthermore, they have a non-singular operator product expansion:

$$
\mathcal{Y}(z) \mathcal{X}(w)=1+\mathcal{O}(z-w)
$$

Since we shall be using some of the results from [40] we shall now give the relation between the normalization conventions used here and those used in [40]. The results of [40] can be found by making the following replacements in our formulæ: ${ }^{2}$

$$
\begin{aligned}
& \beta \rightarrow-\beta / 2, \quad \gamma \rightarrow 2 \gamma, \quad \xi \rightarrow \xi / 2, \quad \eta \rightarrow 2 \eta, \quad \phi \rightarrow \phi, \\
& X^{\mu} \rightarrow X^{\mu}, \quad \psi^{\mu} \rightarrow-i \psi^{\mu} / \sqrt{2}, \quad T_{m} \rightarrow T_{B}, \quad T_{F} \rightarrow T_{F} / 2 \text {. }
\end{aligned}
$$

Next we introduce the 16-component spin fields $S^{\alpha}$ and $S_{\alpha}$ in the matter sector, carrying opposite chirality. We shall use the convention that $e^{-\phi / 2} S_{\alpha}$ and $e^{-3 \phi / 2} S^{\beta}$ are GSO even operators. The relevant operator product involving the spin fields are:

$$
\begin{aligned}
\psi^{\mu}(z) e^{-\phi / 2} S_{\alpha}(w) & =\frac{i}{2}(z-w)^{-1 / 2}\left(\gamma^{\mu}\right)_{\alpha \beta} e^{-\phi / 2} S^{\beta}(w)+\cdots, \\
\psi^{\mu}(z) e^{-\phi / 2} S^{\alpha}(w) & =\frac{i}{2}(z-w)^{-1 / 2}\left(\gamma^{\mu}\right)^{\alpha \beta} e^{-\phi / 2} S_{\beta}(w)+\cdots, \\
e^{-3 \phi / 2} S^{\alpha}(z) e^{-\phi / 2} S_{\beta}(w) & =(z-w)^{-2} \delta_{\beta}^{\alpha} e^{-2 \phi}(w)+\cdots, \\
e^{-\phi / 2} S_{\alpha}(z) e^{-\phi / 2} S_{\beta}(w) & =i(z-w)^{-1}\left(\gamma^{\mu}\right)_{\alpha \beta} e^{-\phi} \psi_{\mu}(w)+\cdots,
\end{aligned}
$$

\footnotetext{
${ }^{2}$ With these replacements, the bosonization rule for $\beta, \gamma$ should take the form $\beta=e^{-\phi} \partial \xi, \gamma=\eta e^{\phi}$. Ref. [40] states the bosonization rules as $\beta=e^{-\phi} \partial \xi, \gamma=e^{\phi} \eta$, but this is inconsistent with the operator product expansion $\gamma(z) \beta(w) \simeq(z-w)^{-1}$ used in [40] if we take $\xi, \eta$ to anti-commute with $e^{ \pm \phi}$.
} 
where the $16 \times 16$ matrices $\gamma_{\alpha \beta}^{\mu}$ satisfy the identities:

$$
\begin{aligned}
& \left(\gamma^{i}\right)^{\alpha \beta}=\left(\gamma^{i}\right)^{\beta \alpha}, \quad\left(\gamma^{i}\right)^{\alpha \beta}=\left(\gamma^{i}\right)_{\alpha \beta}, \quad\left\{\gamma^{i}, \gamma^{j}\right\}=2 \delta_{i j}, \quad \text { for } 1 \leq i \leq 9, \\
& \left(\gamma^{0}\right)^{\alpha \beta}=\delta_{\alpha \beta}, \quad\left(\gamma^{0}\right)_{\alpha \beta}=-\delta_{\alpha \beta} \text {. }
\end{aligned}
$$

These are related to the full ten dimensional gamma matrices $\Gamma^{\mu}$ as follows:

$$
\Gamma^{\mu}=\left(\begin{array}{cc}
0 & \left(\gamma^{\mu}\right)^{\alpha \beta} \\
\left(\gamma^{\mu}\right)_{\alpha \beta} & 0
\end{array}\right)
$$

An explicit choice of such gamma matrices can be found e.g. in appendix A of [44]. It will be understood that when we take product of the $\gamma^{\mu}$ 's, the successive $\gamma^{\mu}$ 's will have their indices alternating between upper and lower indices. Therefore $\left(\gamma^{\mu} \gamma^{\nu}\right)_{\beta}^{\alpha}$ will correspond to $\left(\gamma^{\mu}\right)^{\alpha \delta}\left(\gamma^{\nu}\right)_{\delta \beta}$. With this convention, we have

$$
\left\{\gamma^{\mu}, \gamma^{\nu}\right\}=2 \eta^{\mu \nu} I_{16}
$$

where $I_{16}$ denotes the $16 \times 16$ identity matrix. The consistency of (2.13) with $(2.2)$ can be seen by studying various correlation functions. For example, we have

$$
\left\langle c e^{-\phi} \psi^{\mu}\left(z_{1}\right) c e^{-\phi / 2} S_{\alpha}\left(z_{2}\right) c e^{-\phi / 2} S_{\beta}\left(z_{3}\right)\right\rangle=i K \gamma_{\alpha \beta}^{\mu} / 2,
$$

where $K$ is an overall constant giving $\left\langle c \partial c \partial^{2} c e^{-2 \phi}\right\rangle / 2$ in the holomorphic sector. This can be obtained by either taking the operator product of the second and third operators first using (2.13) and then using (2.2), or by taking the operator product of the first and the second operator first using (2.13) and then using (2.13) again.

We now give the mode expansion of the various fields. The ghost and the matter fields have mode expansions

$$
\begin{aligned}
b(z) & =\sum b_{n} z^{-n-2}, & c(z) & =\sum_{n} c_{n} z^{-n+1}, \\
\beta(z) & =\sum_{n} \beta_{n} z^{-n-\frac{3}{2}}, & \gamma(z) & =\sum_{n} \gamma_{n} z^{-n+\frac{1}{2}}, \\
\eta(z) & =\sum_{n} \eta_{n} z^{-n-1}, & \xi(z) & =\sum_{n} \xi_{n} z^{-n}, \\
i \sqrt{2} \partial X^{\mu}(z) & =\sum_{n} \alpha_{n}^{\mu} z^{-n-1}, & i \sqrt{2} \psi^{\mu}(z) & =\sum_{n} d_{n}^{\mu} z^{-n-1 / 2} .
\end{aligned}
$$

Also useful will be the mode expansions of the total stress tensors of the matter and ghost superconformal field theory and the super-stress tensor of the matter theory:

$$
T(z)=\sum L_{n} z^{-n-2}, \quad T_{F}(z)=\frac{1}{2} \sum_{n} G_{n}^{(m)} z^{-n-3 / 2} .
$$

Note that in this equation $T(z)$ refers to the total stress tensor of all the fields, while $T_{F}$ is the super-stress tensor of the matter fields only. The superscript $(m)$ of $G_{n}^{(m)}$ will serve to remind us of this. 
The normalization conventions described above will be used for both closed and open strings For open strings the expansion coefficients of the anti-holomorphic fields are not independent, but are related to those of the holomorphic fields. For computing correlation functions on the upper half plane, this relationship is used to arrive at the doubling trick in which we replace the upper half plane by the full complex plane and the anti-holomorphic fields in the upper half plane by holomorphic fields at the complex conjugate points.

Finally, we state the normalization of the vacua of the closed string and the open string. For the closed string vacuum carrying momentum $k$, we choose the normalization [41]:

$$
\left\langle k\left|c_{-1} \bar{c}_{-1} c_{0} \bar{c}_{0} c_{1} \bar{c}_{1} e^{-2 \phi}(0) e^{-2 \bar{\phi}}(0)\right| k^{\prime}\right\rangle=-(2 \pi)^{10} \delta^{(10)}\left(k+k^{\prime}\right) .
$$

The normalization of the open string vacuum on a $p$-brane will be chosen as:

$$
\left\langle k\left|c_{-1} c_{0} c_{1} e^{-2 \phi}(0)\right| k^{\prime}\right\rangle=(2 \pi)^{p+1} \delta^{(p+1)}\left(k+k^{\prime}\right) .
$$

\section{Conventions for string field theory}

We shall now review some of the relevant properties of open-closed superstring field theory that describes the coupled dynamics of the degrees of freedom of a D-p-brane and the closed string degrees of freedom. We shall need only a small part of the string field theory and not the full details. The full details can be found in [45, 46], but our convention differs from that of [46] in one important way. In the analysis of [46] the kinetic term of the closed string fields was accompanied by a factor of $g_{s}^{-2}$, that of the open string fields was accompanied by a factor of $g_{s}^{-1}$ and the normalization of the interaction terms were specified only implicitly by requiring that they satisfy appropriate sewing identities. Here we shall accompany the kinetic term of the closed string fields by a constant $\kappa^{-2}$ and that of the open string fields by a different constant $g_{o}^{-1}$ and adjust the relation between $\kappa$ and $g_{o}$ so that the interaction terms have simple normalization. This corresponds to appropriate rescaling of the closed and the open string fields. We shall introduce a third constant $g_{s}$ such that the tension of a BPS D- $p$-brane is given by $(2 \pi)^{-p} / g_{s}$. In particular the type IIB D-instanton action will be given by $2 \pi / g_{s}$.

\subsection{Closed string sector of string field theory}

We shall begin by writing down the kinetic term and the sphere 3-point interaction terms for the NSNS sector classical closed string field. We denote the NSNS sector classical closed string field by a state $\left|\psi_{c}\right\rangle$ in the NSNS sector of the closed string Hilbert space of ghost number 2, satisfying,

$$
\left(b_{0}-\bar{b}_{0}\right)\left|\psi_{c}\right\rangle=0, \quad\left(L_{0}-\bar{L}_{0}\right)\left|\psi_{c}\right\rangle=0,
$$

and write the quadratic and the cubic term in the action as:

$$
S_{c}=\frac{4}{\kappa^{2}}\left(\frac{1}{2}\left\langle\psi_{c}\left|c_{0}^{-}\left(Q_{B}+\bar{Q}_{B}\right)\right| \psi_{c}\right\rangle+\frac{1}{3 !}\left\{\psi_{c}^{3}\right\}\right), \quad c_{0}^{-} \equiv\left(c_{0}-\bar{c}_{0}\right) / 2,
$$

where $\left\{V_{1} V_{2} V_{3}\right\}$ is given by the sphere correlation function of a pair of PCOs and three closed string vertex operators $V_{1}, V_{2}, V_{3}$, inserted using appropriate local coordinate system 
specified by string field theory. The correlation function is computed with the normalization (2.20). Our sign convention for the action is such that in the Euclidean (Lorentzian) theory we take the weight factor in the path integral to be $e^{S}\left(e^{i S}\right)$. The string field $\left|\phi_{c}\right\rangle$ with canonical normalization is related to $\psi_{c}$ via

$$
\left|\psi_{c}\right\rangle=\kappa\left|\phi_{c}\right\rangle
$$

so that

$$
S_{c}=4\left(\frac{1}{2}\left\langle\phi_{c}\left|c_{0}^{-}\left(Q_{B}+\bar{Q}_{B}\right)\right| \phi_{c}\right\rangle+\frac{\kappa}{3 !}\left\{\phi_{c}^{3}\right\}\right), \quad c_{0}^{-} \equiv\left(c_{0}-\bar{c}_{0}\right) / 2,
$$

To check that the kinetic term has the correct normalization, we can fix Siegel gauge $b_{0}\left|\phi_{c}\right\rangle=0$. In this gauge we can replace $Q_{B}+\bar{Q}_{B}$ by $c_{0} L_{0}+\bar{c}_{0} \bar{L}_{0}$, and the kinetic term of the action reduces to:

$$
\left\langle\phi_{c}\left|c_{0} \bar{c}_{0}\left(L_{0}+\bar{L}_{0}\right)\right| \phi_{c}\right\rangle .
$$

Since each of $L_{0}$ and $\bar{L}_{0}$ have additive terms $k^{2} / 4$, the kinetic term has the correct normalization $k^{2} / 2$. In particular, if we define the graviton field $h_{\mu \nu}$ as the following term in the expansion of $\left|\phi_{c}\right\rangle$ :

$$
-\int \frac{d^{10} k}{(2 \pi)^{10}} h_{\mu \nu}(k) c_{1} \bar{c}_{1} d_{-1}^{\mu} \bar{d}_{-1}^{\nu} e^{-\phi}(0) e^{-\bar{\phi}}(0)|k\rangle,
$$

then, with the normalization (2.20), the kinetic term for $h_{\mu \nu}$ will take the form:

$$
-\frac{1}{2} \int \frac{d^{10} k}{(2 \pi)^{10}} h_{\mu \nu}(-k) k^{2} h^{\mu \nu}(k)
$$

This agrees with the quadratic term in the Einstein action,

$$
\frac{1}{2 \kappa^{2}} \int d^{10} x \sqrt{-\operatorname{det} g} R,
$$

in the de Donder gauge, if we expand the metric as

$$
g_{\mu \nu}=\eta_{\mu \nu}+2 \kappa \int \frac{d^{10} k}{(2 \pi)^{10}} h_{\mu \nu}(k) e^{i k \cdot x} .
$$

In this convention, a normalized graviton state of momentum $k$ and polarization $e_{\mu \nu}$ in the $(-1,-1)$ picture has the form:

$$
-e_{\mu \nu} c_{1} \bar{c}_{1} d_{-1}^{\mu} \bar{d}_{-1}^{\nu} e^{-\phi}(0) e^{-\bar{\phi}}(0)|k\rangle, \quad e_{\mu \nu}=e_{\nu \mu}, \quad \eta^{\mu \nu} e_{\mu \nu}=0, \quad k^{\mu} e_{\mu \nu}=0, \quad e^{\mu \nu} e_{\mu \nu}=1 .
$$

Using (2.18), the associated vertex operator is given by

$$
V=-2 e_{\mu \nu} c \bar{c} e^{-\phi} \psi^{\mu} e^{-\bar{\phi}} \bar{\psi}^{\nu} e^{i k . X} .
$$

We shall also need the zero picture vertex operator of this state, obtained by multiplying this by the picture changing operators $\mathcal{X} \overline{\mathcal{X}}$. This takes the form:

$$
2 e_{\mu \nu} \bar{c} c\left\{\partial X^{\mu}+i k_{\rho} \psi^{\rho} \psi^{\mu}\right\}\left\{\bar{\partial} X^{\nu}+i k_{\sigma} \bar{\psi}^{\sigma} \bar{\psi}^{\nu}\right\} e^{i k . X}+\cdots,
$$


where $\cdots$ involves terms proportional to $\gamma \psi^{\mu}$ and $\bar{\gamma} \bar{\psi}^{\nu}$ that will not be needed for our analysis. This agrees with the conventions of [40] after using the translation rules (2.12).

We shall now argue that with this normalization the three point functions of the gravitons also agree with that of [40]. For this let us consider three gravitons with momenta $\left\{k_{i}\right\}$ and polarizations $e_{\mu \nu}^{(i)}$ for $1 \leq i \leq 3$. Let us denote by $V_{i}$ 's their vertex operators. Comparing (3.6) with (3.10) we see that the $V_{i}$ 's are given as in (3.11). It now follows from (3.4) that the three graviton amplitude is given by

$$
4 i \kappa\left\{V_{1} V_{2} V_{3}\right\}=4 i \kappa\left\langle V_{1}\left(z_{1}\right) V_{2}\left(z_{2}\right) V_{3}\left(z_{3}\right)\right\rangle
$$

where \langle\rangle denotes correlation function on the sphere and $z_{1}, z_{2}$ and $z_{3}$ are three fixed points on the sphere. The factor of $i$ is the standard factor that arises in the computation of the S-matrix, taking into account the fact that in Lorentzian signature the path integral is weighted by $e^{i S}$. On the other hand, in the notation of [40], the same amplitude would have been given by

$$
i g_{c}^{3} \frac{8 \pi}{g_{c}^{2}}\left\langle V_{1}\left(z_{1}\right) V_{2}\left(z_{2}\right) V_{3}\left(z_{3}\right)\right\rangle=8 \pi i g_{c}\left\langle V_{1}\left(z_{1}\right) V_{2}\left(z_{2}\right) V_{3}\left(z_{3}\right)\right\rangle, \quad g_{c}=\frac{\kappa}{2 \pi} .
$$

The factor of $g_{c}^{3}$ arises from the convention that each closed string vertex operator is accompanied by a factor of $g_{c}$ and the $8 \pi / g_{c}^{2}$ factor multiplies every sphere amplitude, determined in [40] by the requirement of factorization. The relation $g_{c}=\kappa /(2 \pi)$ was needed to get the correct three graviton coupling as computed from (3.8), (3.9).

We now see that (3.13) and (3.14) agree. Since it was shown in [40] that (3.14) computed with these vertex operators (3.11) agrees with the one computed from the EinsteinHilbert action with gravitational coupling $\kappa$, we conclude that $\kappa$ appearing in (3.2) is the gravitational coupling constant appearing in (3.8). Once the conventions have been matched, it follows that all the higher order amplitudes computed from the action (3.2) also agree with those computed in [40]. We shall now briefly indicate how this works for the four point function. According to (3.2) there will be a contribution to the four point function obtained by joining a pair of three point vertices by a propagator. In the Euclidean theory three point vertices are each proportional to $4 \kappa$ times appropriate three point functions on the sphere, while it follows from (3.5) that the propagator is given by

$$
-\frac{1}{2} \bar{b}_{0} b_{0}\left(L_{0}+\bar{L}_{0}\right)^{-1} \delta_{L_{0}, \bar{L}_{0}}=\frac{1}{2} \frac{1}{2 \pi} b_{0} \bar{b}_{0} \int_{0}^{\infty} d s \int_{0}^{2 \pi} d \theta e^{-s\left(L_{0}+\bar{L}_{0}\right)} e^{i \theta\left(L_{0}-\bar{L}_{0}\right)}
$$

Standard manipulation in conformal field theory now shows that the effect of the exponential factors and sum over all the internal states in the propagator is to sew the two three punctured spheres into a four punctured sphere. The $b_{0}, \bar{b}_{0}$ factors convert one of the unintegrated vertex operators into an integrated vertex operator and the integral over $s$ and $\theta$ generates integration over the location $z$ of the integrated vertex operator with measure $d^{2} z / 2$ where for $z=x+i y, d^{2} z \equiv 2 d x d y$. This has been reviewed in appendix A. Therefore after Wick rotation to Lorentzian signature, we get a net normalization factor:

$$
i(4 \kappa)^{2}\left(\frac{1}{4 \pi}\right) \frac{1}{2}=2 i \kappa^{2} / \pi,
$$


besides the integral over the sphere four point function with three fixed and one integrated vertex operators with measure $d^{2} z$. On the other hand, according to the prescription of [40] the amplitude will get a factor of $g_{c}^{4}$ from the four vertex operators, a factor of $8 \pi / g_{c}^{2}$ from the sphere and the standard factor of $i$ for the S-matrix. This generates a multiplicative factor:

$$
i g_{c}^{4} \times 8 \pi g_{c}^{-2}=i 8 \pi(\kappa /(2 \pi))^{2}=2 i \kappa^{2} / \pi .
$$

This is in agreement with (3.16). This agreement is not surprising, since the normalization of the amplitude was fixed in [40] by demanding that the amplitudes factorize correctly, while in the amplitudes computed from string field theory, the factorization of the amplitude is guaranteed.

This can also be generalized to higher point function. Given an $n$-point function, adding another vertex operator can be achieved by sewing of a three point function using a propagator. From (3.4), the three point function gives a factor of $4 \kappa$, whereas the propagator generates an integral with measure $d^{2} z /(8 \pi)$. Therefore the net effect is multiplication by a factor of $\kappa /(2 \pi)=g_{c}$ and the integration over the location of the puncture with measure $d^{2} z$. This agrees with the prescription of [40].

The Ramond sector of closed string field theory is somewhat more involved, but we shall not need this for our analysis.

\subsection{Open string sector of string field theory}

We now turn to the open string sector of the open-closed string field theory on a $\mathrm{D} p$-brane. The NS sector string field $\left|\psi_{\mathrm{NS}}\right\rangle$ is taken to be a state with picture number -1 in the open string Hilbert space, and the quadratic and cubic terms in the action take the form:

$$
\frac{1}{g_{o}^{2}}\left[\frac{1}{2}\left\langle\psi_{\mathrm{NS}}\left|Q_{B}\right| \psi_{\mathrm{NS}}\right\rangle+\frac{1}{3 !}\left\{\psi_{\mathrm{NS}}^{3}\right\}\right],
$$

where in the definition of $\left\{V_{1} V_{2} V_{3}\right\}$ we include disk amplitudes with one PCO insertion, computed with the standard normalization given in (2.21) and sum over both cyclic ordering of the open string vertex operators $V_{1}, V_{2}, V_{3}$. This explains the factor of $1 / 3$ ! instead of the usual factor of $1 / 3 . g_{o}$ is the open string coupling whose relation to the closed string coupling constant $\kappa$ will be given later. If we define the field $\left|\phi_{\mathrm{NS}}\right\rangle$ via,

$$
\left|\psi_{\mathrm{NS}}\right\rangle=g_{\mathrm{o}}\left|\phi_{\mathrm{NS}}\right\rangle
$$

then, up to this order, the action takes the form

$$
\frac{1}{2}\left\langle\phi_{\mathrm{NS}}\left|Q_{B}\right| \phi_{\mathrm{NS}}\right\rangle+\frac{g_{o}}{3 !}\left\{\phi_{\mathrm{NS}}^{3}\right\}
$$

Since in the Siegel gauge $Q_{B}$ is replaced by $c_{0} L_{0}$, and since $L_{0}$ acting on open string states has an additive term $k^{2}$, the kinetic term has standard normalization. Therefore the 3 -point coupling between three physical open string states is given by $g_{0}$ times the disk 3point function of the vertex operators with the standard normalization (2.21), without any 
additional factor. Furthermore, following analysis similar to the one described for closed strings, one can show that each additional external open string state gives an additional factor of $g_{o}$, and the new vertex operator has to be converted to integrated picture and integrated along the real axis. This agrees with the normalization used in [40]. Therefore the $g_{o}$ appearing in (3.18) agrees with the one used in [40]..$^{3}$

The infinitesimal gauge transformation parameter of the NS sector of the classical open string field theory corresponds to an arbitrary NS sector state $|\theta\rangle$ of ghost number 0 . The gauge transformation law up to order $\phi_{\mathrm{NS}}$ takes the form:

$$
\delta\left|\phi_{\mathrm{NS}}\right\rangle=Q_{B}|\theta\rangle-g_{o}\left[\theta \phi_{\mathrm{NS}}\right],
$$

where $[A B]$ is defined so that for any state $|C\rangle,{ }^{4}$

$$
\langle C \mid[A B]\rangle=\{C A B\} .
$$

Finally we turn to the Ramond sector of the theory. Usually the construction of the kinetic term requires either adding a free field [41] or including a projection operator [49]. However the construction simplifies if we focus on the effective action involving only the zero mass level states, after integrating out all the massive modes. In this case we can take the classical string field to be a state $\left|\psi_{R}\right\rangle$ of the open string of ghost number 1 and picture number $-1 / 2$ and the action up to the cubic order can be taken to be of the form:

$$
S=\frac{1}{g_{o}^{2}}\left[\frac{1}{2}\left\langle\psi_{R}\left|\mathcal{Y}_{0} Q_{B}\right| \psi_{R}\right\rangle+\frac{1}{3 !}\left\{\psi_{R}^{2} \psi_{\mathrm{NS}}\right\}\right],
$$

where $\left\{\psi_{R}^{2} \psi_{\mathrm{NS}}\right\}$ is given by the disk amplitude without any PCO insertion and,

$$
\mathcal{Y}_{0}=\oint \frac{d z}{z} \mathcal{Y}(z), \quad \mathcal{X}_{0}=\oint \frac{d z}{z} \mathcal{X}(z) .
$$

The $\oint$ includes a factor of $1 /(2 \pi)$ so that $\oint d z / z=1$. For the full string field theory this is not an acceptable action since the Hilbert space contains states in the kernel of $\mathcal{Y}_{0}$, but at mass level zero this problem is absent. Defining $\left|\phi_{R}\right\rangle=\left|\psi_{R}\right\rangle / g_{o}$, we can express the action as

$$
\frac{1}{2}\left\langle\phi_{R}\left|\mathcal{Y}_{0} Q_{B}\right| \phi_{R}\right\rangle+\frac{g_{o}}{3 !}\left\{\phi_{R}^{2} \phi_{\mathrm{NS}}\right\}
$$

Note that we have used the same coupling constant $g_{o}$ for the NS and R-sector action. This can be seen from the fact that a four point amplitude of two NS and two R sector states has contribution from a pair of R-R-NS interaction vertices connected by an R-sector propagator and also one R-R-NS and one NS-NS-NS interaction vertex connected by an NS sector propagator. Therefore if we use different coupling constants for the R-R-NS and NS-NS-NS interaction terms, the moduli space integrands of these two contributions to R-R-NS-NS amplitude will not match.

\footnotetext{
${ }^{3}$ One should keep in mind however that the relation between $g_{o}$ and $\kappa$ or $g_{s}$ depends on the value of $p$, i.e. the particular $\mathrm{D} p$-brane we are considering.

${ }^{4}$ When the states $A, B, C$ are not all grassmann odd, the contributions to $\{A B C\}$ from different cyclic orderings come with opposite signs [46], e.g. in Witten's open string field theory [47, 48], $\left[\theta \phi_{\mathrm{NS}}\right]=$ $\theta * \phi_{\mathrm{NS}}-\phi_{\mathrm{NS}} * \theta$.
} 


\subsection{Interaction between open and closed strings}

We shall now describe the normalization of some interaction terms that involve closed strings (and possibly open strings) on Riemann surfaces with boundaries. Since our analysis in sction 6, where these interaction terms will be used, will involve product of four copies of the disk amplitude with identical interaction vertices, the overall sign and factors of $i$ in these interaction terms will not be important and will be ignored. ${ }^{5}$

The elementary interaction term is the one point function of the closed string on the disk. The corresponding term in the action, denoted by $\left\{\psi_{c}\right\}_{D}$ is defined via the relation:

$$
\left\{\psi_{c}\right\}_{D}=\frac{\mathcal{T}}{2}\left\langle\left(c_{0}^{-} \psi_{c}\right)\right\rangle_{D}
$$

where $\mathcal{T}$ is the tension of the $\mathrm{D} p$-brane under consideration, and \langle\rangle$_{D}$ on the right hand side is the closed string one point function on the disk computed with the normalization (2.21). The closed string is inserted at the center of the disk $z=0$ using the local coordinate $e^{\beta} z$, where $z$ is the coordinate system in which the disk is described by $|z| \leq 1$ and $\beta$ is a parameter that characterizes the string field theory under consideration [46]. (3.26) can be taken as the definition of the D-brane tension. It has been shown in appendix B that this definition of the brane tension agrees with the usual definition based on the low energy effective action.

Next we shall describe the interaction term involving disk amplitudes with multiple insertions of closed strings and open strings. In the action it will appear as:

$$
\sum_{m, n} \frac{1}{m ! n !}\left\{\psi_{c}^{m} \psi_{o}^{n}\right\}_{D}=\sum_{m, n} \frac{1}{m ! n !} \kappa^{m} g_{o}^{n}\left\{\phi_{c}^{m} \phi_{o}^{n}\right\}_{D},
$$

where $\psi_{o}$ stands for the open string fields $\psi_{\mathrm{NS}}$ or $\psi_{R}, \phi_{c}=\psi_{c} / \kappa$ and $\phi_{o}=\psi_{o} / g_{o}$ are the canonically normalized fields, and,

$$
\left\{\psi_{c}^{m} \psi_{o}^{n}\right\}_{D}=\pi \mathcal{T} \int\left\langle\psi_{c}^{m} \psi_{o}^{n}\right\rangle_{D}
$$

Here $\left\langle\psi_{c}^{m} \psi_{o}^{n}\right\rangle_{D}$ denotes correlation function on the disk / upper half plane with appropriate number of PCO insertions, computed with the normalization (2.21), with the vertex operators inserted with choice of local coordinates appropriate to the string field theory under consideration and the integral runs over part of the moduli space of the associated Riemann surface with punctures, as prescribed by the particular version of the string field theory we consider. If we use the $\mathrm{SL}(2, \mathrm{R})$ invariance to fix the position of one closed string puncture and one open string puncture, then for the rest of the punctures the integration measure is fixed as follows. For a variable closed string puncture at position $z=x+i y$, the integration measure is taken to be $d^{2} z /(2 \pi)$ where $d^{2} z=2 d x d y$, whereas for a variable

\footnotetext{
${ }^{5}$ If we want to be more careful, we need to include additional factor of $i$ in (3.28) in order to have compatibility with sewing relations. This is related to the fact that for a disk amplitude with closed and open strings, if we make an $\mathrm{SL}(2, R)$ transformation to go from a configuration with one fixed closed string puncture and one fixed open string puncture to one with three fixed open string punctures, the resulting integration measure over the closed string puncture is given by $i d^{2} z$ instead of $d^{2} z$.
} 
open string puncture at position $x$, the integration measure is taken to be $d x$. This is consistent with the normalization of the integration measure over closed string punctures on the sphere and open string punctures on the disk found in sction 3.1 and sction 3.2. The extra factor of $2 \pi$ in (3.28) relative to (3.26) reflects the fact that the disk with one closed string insertion at the origin has a conformal Killing vector that rotates the disk around the origin, and the volume of this group is $2 \pi$. Therefore in the computation of the one point function of closed strings on the disk there is an implicit division by a factor of $2 \pi$ that needs to be removed in (3.28).

With this normalizations, we can check iteratively that the interaction terms will satisfy the appropriate sewing relations needed for the gauge invariance of the theory. For example, let us consider a disk amplitude with $m$ on-shell closed strings and $n$ on-shell open strings with canonically normalized external states. Part of this contribution comes from a Feynman diagram where a closed string three point vertex with two external states is connected to a disk amplitude with $m-1$ closed strings and $n$ open strings by a closed string propagator. In this case we get a factor of $1 /(4 \pi)$ from the propagator (3.15), and another factor of $1 / 2$ while writing $d s d \theta$ in terms of $d^{2} z$ as discussed above (3.16) and in appendix A. Therefore the amplitude involves a factor of $4 \kappa$ from the closed string three point vertex as given in (3.13), a factor of $\pi \mathcal{T} \kappa^{m-1} g_{o}^{n}$ from the disk amplitude with $(m-1)$ closed string and $n$ open strings, a factor of $(2 \pi)^{-(m-2)}$ associated with the integration measure of the $(m-2)$ integrated closed string puncture on the disk and a factor of $1 /(8 \pi)$ from the closed string propagator. This gives a net factor of $\mathcal{T} \kappa^{m} g_{o}^{n} / 2 \times(2 \pi)^{-(m-2)}$ accompanying this diagram. On the other hand, the same amplitude also gets a contribution from the interaction vertex (3.27) with $m$ external closed strings and $n$ external open strings, covering a different region of the moduli space. The associated normalization factor is $\pi \mathcal{T} \kappa^{m} g_{o}^{n}$ times $(2 \pi)^{-(m-1)}$ since there are $(m-1)$ integrated closed string punctures on the disk with $m$ closed string punctures. Therefore the two normalization factors match, as required by gauge invariance. A similar analysis involving sewing via an open string propagator connecting a disk amplitude with $m$ closed strings and $(n-1)$ open strings and the disk amplitude with three open strings can be used to check consistency of the relative normalization given in (3.28) for $(m, n)$ and $(m, n-1)$.

\subsection{Relation between the different coupling constants}

We are now in a position to discuss the relation between $\kappa, g_{o}$ and $\mathcal{T}$. In the following we shall ignore factors of $i$ and minus signs in the intermediate steps since $\kappa, g_{o}$ and $\mathcal{T}$ are all positive. The relation between $g_{o}$ and $\mathcal{T}$ may be found as follows. Let us consider a disk amplitude with $m$ on-shell closed strings and $n$ on-shell open strings with canonically normalized external states. Part of this contribution comes from the interaction vertex (3.28) with $m$ closed strings and $n$ open strings, with associated normalization factor $\pi \mathcal{T} \kappa^{m} g_{o}^{n}$ times $(2 \pi)^{-(m-1)}$. We shall write this as $\mathcal{T} \kappa^{m} g_{o}^{n} / 2 \times(2 \pi)^{-(m-2)}$. On the other hand, the same amplitude receives contribution from another class of Feynman diagrams in which a disk amplitude with $p$ closed string states and $q$ open string states is joined to another disk amplitude with $m-p$ closed string states and $n-q+2$ open string states by an open string propagator. In this case this amplitude gets a factor of $\pi \mathcal{T} \kappa^{p} g_{o}^{q} \times(2 \pi)^{-(p-1)}$ 
and $\pi \mathcal{T} \kappa^{m-p} g_{o}^{n-q+2} \times(2 \pi)^{-(m-p-1)}$ from the two interaction vertices. The Siegel gauge open string propagator

$$
b_{0}\left(L_{0}\right)^{-1}=b_{0} \int_{0}^{\infty} e^{-s L_{0}},
$$

does not generate any extra factor. This gives a net factor of $\pi^{2} \mathcal{T}^{2} \kappa^{m} g_{o}^{n+2} \times(2 \pi)^{-(m-2)}$. Equating the two factors associated with the two Feynman diagrams we get $\mathcal{T} \kappa^{m} g_{o}^{n} / 2=$ $\pi^{2} \mathcal{T}^{2} \kappa^{m} g_{o}^{n+2}$. This gives

$$
\mathcal{T}=\frac{1}{2 \pi^{2} g_{o}^{2}}
$$

This agrees with the result of [50] obtained by different method and also with the result of [40]. For D-instantons we shall label $\mathcal{T}$ as

$$
\mathcal{T}=\frac{2 \pi}{g_{s}} .
$$

Therefore, we have

$$
g_{o}^{2}=g_{s} /\left(4 \pi^{3}\right) .
$$

$g_{s}$ is a useful parameter since $\tau=a+i g_{s}^{-1}$, where $a$ is the vacuum expectation value of the Ramond-Ramond scalar, transforms as $\tau \rightarrow-1 / \tau$ under S-duality transformation.

The relation between $\kappa$ and $\mathcal{T}$ can be found by considering the annulus zero point function. On the one hand, this can be obtained by joining a pair of disk one point function of closed strings by a closed string propagator. Since the disk one point function of canonically normalized closed string is proportional to $\kappa \mathcal{T}$, this contribution will be proportional to $(\kappa \mathcal{T})^{2}$. On the other hand this contribution may be expressed as an integral of the open string partition function that does not depend on any parameter. Equating these two expressions we can determine $\kappa \mathcal{T}$. This computation was carried out in [40] and since our conventions for the parameters agree with that of [40] we just state the result:

$$
\kappa^{2} \mathcal{T}^{2}=\frac{1}{2}(2 \pi)^{7-2 p} .
$$

For D-instantons $p=-1$ and $\mathcal{T}=2 \pi / g_{s}$. This gives

$$
\kappa^{2}=2^{6} \pi^{7} g_{s}^{2} .
$$

\section{Normalization of the D-instanton amplitude}

The general expression for the contribution to an amplitude due to a single D-instanton in type IIB string theory, with action $2 \pi / g_{s}$, takes the form

$$
\mathcal{N} e^{-2 \pi / g_{s}} \mathcal{A},
$$

where $\mathcal{N}$ is a normalization constant and $\mathcal{A}$ is the usual world-sheet contribution to the amplitude. We have not explicitly written down the $e^{2 \pi i a}$ factor since we have not switched on RR scalar background, but the presence of this factor follows from general considerations. Our goal in this section will be to compute $\mathcal{N}$. As mentioned below (1.2), we shall not be careful about the overall phase of $\mathcal{N}$ since it can be absorbed into a shift of $a$. 


\subsection{Annulus partition function}

The general procedure for computing the normalization of the D-instanton amplitude was described in [31]. As in [31], we shall formally write the normalization $\mathcal{N}$ as:

$$
\mathcal{N}=i \zeta e^{A}
$$

Here $\zeta$ is a possible multiplier factor that specifies what multiple of the full steepest descent contour of the D-instanton is included in the actual integration contour over the string fields. This will be analyzed in sction 5. The factor of $i$ is common to all string amplitudes and reflects the usual factor of $i$ that appears while relating the analytic continuation of the Euclidean momentum space Green's functions to the S-matrix via the LSZ prescription. A is the annulus partition function, formally written as [40]

$$
A=\int_{0}^{\infty} \frac{d t}{2 t}\left[\frac{1}{2} \eta(i t)^{-12}\left\{\vartheta_{3}(0 \mid i t)^{4}-\vartheta_{4}(0 \mid i t)^{4}-\vartheta_{2}(0 \mid i t)^{4}+\vartheta_{1}(0 \mid i t)^{4}\right\}\right]
$$

where the $\vartheta_{i}$ 's are the Jacobi theta functions and $\eta$ is the Dedekind $\eta$ function. The coefficient of $e^{-2 \pi n t}$ inside the square bracket counts the difference between the bosonic and fermionic open string states on the D-instanton with $L_{0}$ eigenvalue $n$. The first two terms inside the square bracket reflect the contribution from the NS sector states and the last two terms reflect the contribution from the $\mathrm{R}$ sector states. The last term is actually zero, but we have written it here since this is the form in which it arises when we take the trace over open string states. The $1 / 2$ inside the square bracket comes from the GSO projection operator $\left(1+(-1)^{f}\right) / 2$ where $f$ is the world-sheet fermion number.

Now the annulus partition function $A$ given in (4.3) actually vanishes due to cancellation between the NS and R sector states. However this cancellation cannot be trusted since the $L_{0}=0$ sector represents NS and R sector zero modes for which (4.3) is not applicable. Nevertheless the cancellation in the $L_{0}>0$ sector shows that the contribution to $\mathcal{N}$ comes entirely from the zero mode sector. Our strategy, following [31], will be to represent the zero mode contribution to $\mathcal{N}$ as integrals over the zero mode string fields, and then explicitly carry out these integrals.

To proceed further, it will be useful to regulate the contribution from the $L_{0}=0$ states to (4.3) by giving a small positive value to $L_{0}$. This can be achieved for example by considering open strings stretched from one D-instanton to a neighboring D-instanton separated by a small distance $a$ and noting that in the limit of zero separation the spectrum reduces to that of open strings with two ends lying on the same D-instanton. For non-zero separation between the two D-instantons, both the NS and the R-sector modes get a small positive contribution to $L_{0}$ given by $h=a^{2} /\left(4 \pi^{2}\right)$, introducing an additional multiplicative factor $e^{-2 \pi t h}$ in the integrand. Noting that the term inside the square bracket in (4.3) gets a contribution of 8 each from the NS and the R-sector zero modes, we can express the regulated zero mode contribution to (4.2) as:

$$
\mathcal{N}=i \zeta \exp \left[\int_{0}^{\infty} \frac{d t}{2 t}\left(8 e^{-2 \pi t h}-8 e^{-2 \pi t h}\right)\right]
$$


We can now use the general result ${ }^{6}$

$$
\int_{0}^{\infty} \frac{d t}{2 t}\left[\sum_{i=1}^{n} e^{-2 \pi t h_{i}^{b}}-\sum_{i=1}^{n} e^{-2 \pi t h_{i}^{f}}\right]=\frac{1}{2} \ln \frac{\prod_{i=1}^{n} h_{i}^{f}}{\prod_{i=1}^{n} h_{i}^{b}}
$$

to express $\mathcal{N}$ as

$$
\mathcal{N}=i \zeta \sqrt{\frac{h^{8}}{h^{8}}}
$$

For reasons that will be clear soon, we shall express this as an integral of the form:

$$
\mathcal{N}=i \zeta \int\left\{\prod_{\mu=0}^{9} \frac{d \xi_{\mu}}{\sqrt{2 \pi}}\right\} d p d q \exp \left[-\frac{1}{2} h \sum_{\mu=0}^{9} \xi_{\mu} \xi^{\mu}-h p q\right] \int \prod_{\alpha=1}^{16} d \chi_{\alpha} \exp \left[\frac{1}{2} g_{\alpha \beta} \chi_{\alpha} \chi_{\beta}\right]
$$

where $\xi_{\mu}$ are grassmann even modes, $p, q$ are grassmann odd modes, $\chi_{\alpha}$ are grassmann odd modes and $g_{\alpha \beta}$ is an anti-symmetric, $16 \times 16$ hermitian matrix with the property:

$$
g^{2}=h I_{16},
$$

where $I_{16}$ is the $16 \times 16$ identity matrix. Note that even though we have written the quadratic term in $\xi^{\mu}$ as $\xi_{\mu} \xi^{\mu}$, in euclidean signature this is just $\sum_{\mu}\left(\xi^{\mu}\right)^{2}$ and the integral over the $\xi^{\mu}$ 's is well-defined.

We shall now proceed as follows.

1. First we shall show that (4.7) may be interpreted as the Siegel gauge fixed path integral of the open string field theory on the D-instanton with appropriate normalization. Up to normalization, the modes $\xi^{\mu}$ will represent the translation modes of the D-instanton in the $h \rightarrow 0$ limit, the modes $p$ and $q$ will represent Faddeev-Popov ghosts in the NS sector and the modes $\chi_{\alpha}$ will represent the fermionic collective modes on the D-instanton in the $h \rightarrow 0$ limit.

2. Then we shall show that the Siegel gauge becomes singular in the $h \rightarrow 0$ limit, and this is the reason why the coefficient of the $p q$ term, representing the ghost kinetic operator, vanishes. The remedy will be to work with the original gauge invariant path integral before gauge fixing.

3. We shall integrate over the collective modes at the end following standard procedure. In particular we shall determine the correct normalization factor that relates the modes $\xi^{\mu}$ to the locations $\widetilde{\xi}^{\mu}$ of the D-instanton in Euclidean space time. The integration over the $\widetilde{\xi}^{\mu}$ 's will then generate the standard energy momentum conserving delta function for the momenta of external states entering the amplitude $\mathcal{A}$ in (4.1). The integration over the modes $\chi_{\alpha}$ will force us to insert the vertex operators of each of the sixteen $\chi_{\alpha}$ 's into the world-sheet defining the amplitude $\mathcal{A}$, since otherwise the integral will vanish.

\footnotetext{
${ }^{6}$ To arrive at (4.5) we need to put a lower cut-off $\epsilon$ on the $t$ integral and take the $\epsilon \rightarrow 0$ limit at the end of the calculation. A discussion on this may be found in sction 8 and [51].
} 


\subsection{Gauge invariant string field theory in the $L_{0}=0$ sector}

Since open strings living on the D-instanton do not carry any continuous momenta, the associated open string field theory is zero dimensional, containing a discrete set of modes. Since we shall be working with only the $L_{0}=0$ sector, we begin by listing the basis states in this sector. ${ }^{7}$

$$
\begin{array}{lll}
\mathrm{NS}: & \beta_{-1 / 2} c_{1}|-1\rangle, \quad c_{1} d_{-1 / 2}^{\mu}|-1\rangle, \quad \beta_{-1 / 2} c_{0} c_{1}|-1\rangle, \\
& \gamma_{-1 / 2} c_{1}|-1\rangle, \quad c_{0} c_{1} d_{-1 / 2}^{\mu}|-1\rangle, \quad \gamma_{-1 / 2} c_{0} c_{1}|-1\rangle, \\
\mathrm{R}: \quad & \left(\gamma_{0}\right)^{n} c_{1}|-1 / 2, \alpha\rangle, \quad\left(\gamma_{0}\right)^{n} c_{0} c_{1}|-1 / 2, \alpha\rangle,
\end{array}
$$

where we have defined,

$$
|-1\rangle \equiv e^{-\phi}(0)|0\rangle, \quad|-1 / 2, \alpha\rangle=e^{-\phi / 2} S_{\alpha}(0)|0\rangle .
$$

Since classical open string fields carry ghost number 1, we have the following expansion of the classical fields $\left|\phi_{\mathrm{NS}}\right\rangle$ and $\left|\phi_{R}\right\rangle$ introduced in (3.2):

$$
\begin{aligned}
\left|\phi_{\mathrm{NS}}\right\rangle & =i \phi^{1} \beta_{-1 / 2} c_{0} c_{1}|-1\rangle+\xi_{\mu} c_{1} d_{-1 / 2}^{\mu}|-1\rangle, \\
\left|\phi_{R}\right\rangle & =\chi_{\alpha} c_{1}|-1 / 2, \alpha\rangle,
\end{aligned}
$$

where $\phi^{1}$ and $\xi_{\mu}$ are grassmann even modes and $\chi_{\alpha}$ for $1 \leq \alpha \leq 16$ are grassmann odd modes. The $i$ multiplying the coefficient $\phi^{1}$ reflects the fact that the reality condition on the string field requires an expansion of the form given in (4.12) with real $\phi^{1}$ [52]. As discussed in sction 3.2, the kinetic term of the action is given by:

$$
S=S_{\mathrm{NS}}+S_{R}, \quad S_{\mathrm{NS}}=\frac{1}{2}\left\langle\phi_{\mathrm{NS}}\left|Q_{B}\right| \phi_{\mathrm{NS}}\right\rangle, \quad S_{R}=\frac{1}{2}\left\langle\phi_{R}\left|\mathcal{Y}_{0} Q_{B}\right| \phi_{R}\right\rangle .
$$

Since gauge transformation parameters are described by states of ghost number 0 , we see from (4.10) that there is no gauge transformation parameter in the R sector, while the NS sector contains a single gauge transformation parameters $\theta$ :

$$
\left|\theta_{\mathrm{NS}}\right\rangle=i \theta \beta_{-1 / 2} c_{1}|-1\rangle,
$$

where again the factor of $i$ reflects that real gauge transformation parameter in string field theory corresponds to real $\theta$. Classical gauge transformation law

$$
\delta\left|\phi_{\mathrm{NS}}\right\rangle=Q_{B}\left|\theta_{\mathrm{NS}}\right\rangle,
$$

translates to the following transformation of $\phi^{1}$ and $\xi^{\mu}$ :

$$
\delta \phi^{1}=\theta\left\langle-1\left|\gamma_{1 / 2} c_{-1} Q_{B} \beta_{-1 / 2} c_{1}\right|-1\right\rangle, \quad \delta \xi^{\mu}=i \theta\left\langle-1\left|d_{1}^{\mu} c_{-1} c_{0} Q_{B} \beta_{-1 / 2} c_{1}\right|-1\right\rangle .
$$

The partition function of the theory may now be defined as:

$$
I=\int\left\{\prod_{\mu=0}^{9} d \xi^{\mu}\right\} d \phi^{1}\left\{\prod_{\alpha=1}^{16} d \chi_{\alpha}\right\} e^{S} / \int d \theta .
$$

At this stage the overall normalization of the partition function has been chosen arbitrarily. The final result will be independent of this choice.

\footnotetext{
${ }^{7}$ Note that in the regulated version, what we refer to as $L_{0}=0$ states actually have $L_{0}=h$.
} 


\subsection{Gauge fixing to Siegel gauge}

We now consider the Siegel gauge in the NS sector:

$$
b_{0}\left|\phi_{\mathrm{NS}}\right\rangle=0 .
$$

This translates to:

$$
\phi^{1}=0 .
$$

Using (4.17) we see that the corresponding Faddeev-Popov determinant is given by:

$$
\left\langle-1\left|\gamma_{1 / 2} c_{-1} Q_{B} \beta_{-1 / 2} c_{1}\right|-1\right\rangle \text {. }
$$

This may be represented by introducing a pair of grassmann odd ghost fields $p, q$ defined via,

$$
\left|\phi_{\text {ghost }}\right\rangle=-p \gamma_{-1 / 2} c_{1}|-1\rangle+q \beta_{-1 / 2} c_{1}|-1\rangle \text {, }
$$

with action,

$$
S_{\text {ghost }}=\frac{1}{2}\left\langle\phi_{\text {ghost }}\left|Q_{B}\right| \phi_{\text {ghost }}\right\rangle=-p q\left\langle-1\left|\gamma_{1 / 2} c_{-1} Q_{B} \beta_{-1 / 2} c_{1}\right|-1\right\rangle,
$$

so that $\int d p d q e^{S_{\text {ghost }}}$ gives us the Faddeev-Popov determinant (4.21).

Since the Siegel gauge NS sector field and the ghost field (4.22) both satisfy the Siegel gauge condition $b_{0}|\psi\rangle=0$, the BRST operator $Q_{B}$ reduces to $c_{0} L_{0}$. Therefore the sum of the classical action and the ghost action now takes the form:

$$
S_{\mathrm{NS}}+S_{\text {ghost }}=-\frac{1}{2} \sum_{\mu=0}^{9} h \xi^{\mu} \xi_{\mu}-h p q,
$$

where we have used the fact that the $L_{0}$ eigenvalues of these states is given by $h$. On the other hand, using the form of $\mathcal{Y}$ given in (2.10), and of $Q_{B}$ given in (2.7), (2.8), and the fact that we need total $\phi$-charge -2 to get a non-vanishing disk correlation function, we see that the Ramond action $S_{R}$ given in (4.14) gets contribution only from the $\gamma T_{F}=\eta e^{\phi} T_{F}$ term in $Q_{B}$. Using the expansion (2.19) we may express the $\mathrm{R}$ sector kinetic term as

$$
S_{R}=\frac{1}{2} g_{\alpha \beta} \chi_{\alpha} \chi_{\beta}, \quad g_{\alpha \beta}=\left\langle-1 / 2, \alpha\left|c_{-1} c_{0} G_{0}^{(m)} c_{1}\right|-3 / 2, \beta\right\rangle .
$$

There is a comment that is in order here. If we regularize the path integral over zero modes by considering open strings stretched between a pair of D-instantons, then the string field theory action naturally pairs strings of opposite orientation. This necessarily doubles the spectrum of the theory. In the NS sector we can avoid this problem by working with states with Chan-Paton factors $\sigma_{1}$ or $\sigma_{2}$, since $Q_{B}$ will not mix these sectors. However for the fermions, the operator $G_{0}^{(m)}$ will still pair the states in these two sectors, since $G_{0}^{(m)}$ is linear in the perturbation that separates the D-instantons and this perturbation is proportional to $\sigma_{3}$. Therefore if we just pick states in the sector $\sigma_{1}$ or $\sigma_{2}$ then the kinetic term will vanish. This can be avoided as follows. Let us suppose that we have separated the instantons along the $x^{1}$ direction. In that case it follows from (2.3) and (2.19) that 
acting on the R sector ground state, $G_{0}^{(m)}$ will be proportional to $\gamma^{1}$, which has non-zero matrix element between the dotted and undotted spinors of the $\mathrm{SO}(8)$ group that acts on the coordinates $1, \cdots, 8$. If we now pick the dotted spinors of $\mathrm{SO}(8)$ from the sector with Chan-Paton factor $\sigma_{1}$ and the undotted spinors of $\mathrm{SO}(8)$ from the sector with ChanPaton factor $\sigma_{2}$, then $G_{0}^{(m)}$ will have non-zero matrix element between these states and will provide an action of the form given in (4.25). This of course leaves the phase of the partition function ambiguous, but as mentioned below (1.2), this phase can be absorbed into a redefinition of the vacuum expectation value $a$ of the RR scalar field.

Now using (2.19) and (2.4) we see that

$$
\left\{G_{0}^{(m)}, G_{0}^{(m)}\right\}=2\left(L_{0}^{(m)}-\frac{5}{8}\right) .
$$

On the other hand $c_{1}|-3 / 2, \beta\rangle$ has $L_{0}^{\text {ghost }}=-5 / 8$. Therefore (4.26) gives,

$$
\left(G_{0}^{(m)}\right)^{2} c_{1}|-3 / 2, \beta\rangle=L_{0} c_{1}|-3 / 2, \beta\rangle=h c_{1}|-3 / 2, \beta\rangle .
$$

This in turn shows that the matrix $g_{\alpha \beta}$ defined in (4.25) squares to $h$ times the $16 \times 16$ identity matrix.

After gauge fixing, the partition function $I$ defined in (4.18) takes the form:

$$
\begin{aligned}
I & =\int\left\{\prod_{\mu} d \xi^{\mu}\right\} d p d q\left\{\prod_{\alpha} d \chi_{\alpha}\right\} e^{S+S_{\text {ghost }}} \\
& =\int\left\{\prod_{\mu} d \xi^{\mu}\right\} d p d q\left\{\prod_{\alpha} d \chi_{\alpha}\right\} e^{-\frac{1}{2} \sum_{\mu=0}^{9} h \xi^{\mu} \xi_{\mu}-h p q+\frac{1}{2} g_{\alpha \beta} \chi_{\alpha} \chi_{\beta}} .
\end{aligned}
$$

Comparing this with (4.7), we get,

$$
\mathcal{N}=i \zeta(2 \pi)^{-5} I
$$

Let us now set $h=0$. Since $g_{\alpha \beta}$ squares to $h$ times the identity matrix, $g_{\alpha \beta}$ also vanishes. In this case the action vanishes identically and the integrand becomes independent of $\left\{\xi_{\mu}\right\},\left\{\chi_{\alpha}\right\}$ and $p, q$. Lack of dependence on $\left\{\xi_{\mu}\right\}$ and $\left\{\chi_{\alpha}\right\}$ may be traced to the fact that these are bosonic and fermionic collective modes of the D-instanton, but the lack of dependence on $p$ and $q$ indicates the vanishing of the Faddeev-Popov determinant and therefore the breakdown of the Siegel gauge choice.

\subsection{Gauge invariant partition function}

We circumvent the problem of breakdown of Siegel gauge by replacing $I$ in (4.29) by the original gauge invariant expression (4.18). This gives,

$$
\mathcal{N}=i \zeta(2 \pi)^{-5} \int\left\{\prod_{\mu} d \xi^{\mu}\right\} d \phi^{1}\left\{\prod_{\alpha} d \chi_{\alpha}\right\} e^{S} / \int d \theta .
$$

We shall now set $h=0$ and regard $\xi^{\mu}, \phi^{1}$ and $\chi_{\alpha}$ as degrees of freedom of the open string with both ends lying on the same D-instanton. Substituting (4.12) and (4.13) into (4.14), we get

$$
S=-\frac{1}{4}\left(\phi^{1}\right)^{2}
$$


We can now carry out the $\phi^{1}$ integral, generating a factor of $2 \sqrt{\pi}$. This gives,

$$
\mathcal{N}=i \zeta(2 \pi)^{-5} 2 \sqrt{\pi} \int\left\{\prod_{\mu} d \xi^{\mu}\right\}\left\{\prod_{\alpha} d \chi_{\alpha}\right\} / \int d \theta .
$$

It is to be understood that even though we have written the $\xi^{\mu}$ and $\chi_{\alpha}$ integrals as part of $\mathcal{N}$, these integrals need to be performed after taking the product of $\mathcal{N}$ with the rest of the world-sheet amplitude $\mathcal{A}$ appearing in (4.1).

Our next task is to find the relation between $\xi^{\mu}$ and the D-instanton locations $\widetilde{\xi}^{\mu}$ along the Euclidean space-time. This analysis proceeds as in [31]. We note that the integrated, zero picture vertex operators associated with the mode $\xi^{\mu}$ is given by

$$
\mathcal{X}(z) i \sqrt{2} \psi^{\mu} e^{-\phi}(z)=i \sqrt{2} \partial X^{\mu}(z) .
$$

Now consider the effect of inserting the field $\xi^{\mu}$ into a disk amplitude of open and closed strings with the closed strings carrying total momentum $p^{\mu}$. It follows from (3.28) and (4.33) that this will insert a vertex operator

$$
\int d z g_{o} \xi_{\mu} i \sqrt{2} \partial X^{\mu}(z)
$$

with the integral running along the boundary of the disk. The factor of $g_{o}$ arises from the relation $\left|\psi_{o}\right\rangle=g_{o}\left|\phi_{o}\right\rangle$ and that $\xi_{\mu}$ appears in (4.12) as coefficients in the expansion of field $\left|\phi_{\mathrm{NS}}\right\rangle$ with canonically normalized kinetic term. Using the operator product expansion

$$
\partial X^{\mu}(z) e^{i p_{i} \cdot X\left(z_{i}\right)}=-\frac{i p_{i}^{\mu}}{2\left(z-z_{i}\right)} e^{i p_{i} \cdot X\left(z_{i}\right)}
$$

we can now evaluate the integration over $z$ and get a factor of

$$
g_{o} \xi_{\mu} i \sqrt{2} 2 \pi i\left(-\frac{i}{2} \sum_{i} p_{i}^{\mu}\right)=i g_{o} \pi \sqrt{2} \xi_{\mu}\left(\sum_{i} p_{i}^{\mu}\right),
$$

multiplying the original amplitude without $\xi^{\mu}$ insertion. On the other hand if $\widetilde{\xi}^{\mu}$ denotes the D-instanton location, then the dependence on $\widetilde{\xi}^{\mu}$ of the amplitude is expected to be via a multiplicative factor of the form,

$$
e^{i \widetilde{\xi}_{\mu}\left(\sum_{i} p_{i}^{\mu}\right)}=1+i \widetilde{\xi}_{\mu}\left(\sum_{i} p_{i}^{\mu}\right)+\cdots
$$

Comparing (4.36) with (4.37) we get,

$$
g_{o} \pi \sqrt{2} \xi_{\mu}=\widetilde{\xi}_{\mu}
$$

This gives

$$
\prod_{\mu=0}^{9} d \xi_{\mu}=g_{o}^{-10} \pi^{-10} 2^{-5} \prod_{\mu=0}^{9} d \widetilde{\xi}_{\mu}
$$


By virtue of (4.37), the integration over $\left\{\widetilde{\xi}_{\mu}\right\}$ will generate the momentum conserving delta function $(2 \pi)^{10} \delta^{(10)}\left(\sum_{p} p_{i}\right)$, keeping in mind that these integrals have to be performed after multiplying $\mathcal{N}$ by the rest of the world-sheet amplitude $\mathcal{A}$ as given in (4.1). Therefore, for now we leave the $\widetilde{\xi}^{\mu}$ 's unintegrated and write

$$
\mathcal{N}=i \zeta g_{o}^{-10} \pi^{-10} 2^{-5}(2 \pi)^{-5} 2 \sqrt{\pi} \int\left\{\prod_{\mu} d \widetilde{\xi}_{\mu}\right\}\left\{\prod_{\alpha} d \chi_{\alpha}\right\} / \int d \theta .
$$

Next we shall analyze the result of integration over $\theta$. As in the case of [31], $\theta$ is related to the rigid gauge transformation parameter $\widetilde{\theta}$ under which an open string connecting the D-instanton under study to a second spectator D-instanton picks up a factor of $e^{i \widetilde{\theta}}$. Let us express the NS sector open string field $\left|\widehat{\phi}_{\mathrm{NS}}\right\rangle$ associated with the open string connecting the two instantons by an expansion similar to (4.12), but with the coefficients denoted by $\widehat{\xi}^{\mu}$ and $\widehat{\phi}^{1}$. This will carry a Chan-Paton factor $\left(\begin{array}{ll}0 & 1 \\ 0 & 0\end{array}\right)$. Then according to (3.21), under the gauge transformation generated by $\theta$, the transformation of $\left|\widehat{\phi}_{\mathrm{NS}}\right\rangle$ is given by,

$$
\delta\left|\widehat{\phi}_{\mathrm{NS}}\right\rangle=-g_{\mathrm{o}}\left[\theta \widehat{\phi}_{\mathrm{NS}}\right] .
$$

In particular the transformation law of $\widehat{\xi}^{\mu}$ may be obtained by taking the inner product of this with the state $c_{1} c_{0} d_{-1}^{\mu}|-1\rangle$ with Chan-Paton factor $\left(\begin{array}{ll}0 & 0 \\ 1 & 0\end{array}\right)$. This gives, up to a sign,

$$
\delta \widehat{\xi}^{\mu}=g_{o}\left\{\left(c_{1} c_{0} d_{-1}^{\mu}|-1\rangle\right)\left(i \theta \beta_{-1 / 2} c_{1}|-1\rangle\right)\left(\widehat{\xi}_{\nu} c_{1} d_{-1}^{\nu}|-1\rangle\right\}\right.
$$

The trace over the Chan-Paton factors ensures that only one of the cyclic ordering contributes to the three point function on the disk that defines the \{\} in the above equation. There is one PCO inside this correlation function. Taking its location to coincide with the vertex operator $c \partial \xi e^{-2 \phi}$ of the state $\beta_{-1 / 2} c_{1}|-1\rangle$ multiplying the gauge transformation parameter $\theta$, we can convert the vertex operator of the gauge transformation parameter to:

$$
\mathcal{X}(z) c \partial \xi e^{-2 \phi}(z)=\frac{1}{2} I
$$

where $I$ is the identity operator. Therefore we have

$$
\delta \widehat{\xi}^{\mu}=g_{o} i \theta \frac{1}{2} \widehat{\xi}_{\nu}\left\langle\left(i \sqrt{2} c \partial c \psi^{\mu} e^{-\phi}\left(z_{1}\right)\right)\left(i \sqrt{2} c \psi^{\nu} e^{-\phi}\left(z_{2}\right)\right)\right\rangle_{D}=\frac{i}{2} g_{o} \theta \widehat{\xi}^{\mu}
$$

Comparing this with the infinitesimal rigid $\mathrm{U}(1)$ transformation $\delta \widehat{\xi}^{\mu}=i \widetilde{\theta} \xi^{\mu}$, we get $\theta=$ $2 \widetilde{\theta} / g_{o}$. Since $\widetilde{\theta}$ has period $2 \pi$, this gives,

$$
\int d \theta=4 \pi / g_{o}
$$

Substituting this into (4.40) we get

$$
\mathcal{N}=i \zeta g_{o}^{-10} \pi^{-10} 2^{-5}(2 \pi)^{-5} 2 \sqrt{\pi} \frac{g_{o}}{4 \pi} \int\left\{\prod_{\mu} d \widetilde{\xi}_{\mu}\right\}\left\{\prod_{\alpha} d \chi_{\alpha}\right\} .
$$

Finally note that the variables $\chi_{\alpha}$ are the coefficients of expansion of the field $\left|\phi_{R}\right\rangle$. It will be useful to express $\mathcal{N}$ as integration over the coefficients of expansion of the field $\left|\psi_{R}\right\rangle$ 
since this enters the interaction terms (3.28) without any additional factor of $g_{o}$. To this end we introduce the variables $\tilde{\chi}_{\alpha}$ via

$$
\left|\psi_{R}\right\rangle=\tilde{\chi}_{\alpha} c_{1}|-1 / 2, \alpha\rangle,
$$

Comparing this with (4.13) and using $\left|\psi_{R}\right\rangle=g_{o}\left|\phi_{R}\right\rangle$, we get

$$
\tilde{\chi}_{\alpha}=g_{o} \chi_{\alpha}, \quad \prod_{\alpha} d \chi_{\alpha}=g_{o}^{16} \prod_{\alpha} d \widetilde{\chi}_{\alpha}
$$

since $\chi_{\alpha}$ are grassmann odd variables. Substituting this into (4.46) we get

$$
\mathcal{N}=i \zeta g_{o}^{7} 2^{-11} \pi^{-31 / 2} \int\left\{\prod_{\mu} d \widetilde{\xi}_{\mu}\right\}\left\{\prod_{\alpha} d \widetilde{\chi}_{\alpha}\right\} .
$$

Finally we use (3.32) to express this as: ${ }^{8}$

$$
\mathcal{N}=\mathcal{N}_{0} \int\left\{\prod_{\mu} d \widetilde{\xi}_{\mu}\right\}\left\{\prod_{\alpha} d \widetilde{\chi}_{\alpha}\right\}, \quad \mathcal{N}_{0}=i \zeta g_{s}^{7 / 2} 2^{-18} \pi^{-26}
$$

Integration over the grassmann odd variables shows that unless the rest of the amplitude contains insertions of the $16 \widetilde{\chi}_{\alpha}{ }^{\text {'s, }}$, the result vanishes identically. This will be discussed in sction 6 .

\section{The multiplier factor}

There are two steps involved in the evaluation of the contribution due to a given instanton to the amplitude. The first is to evaluate the contribution to the integral from the steepest descent contour / Lefschetz thimble associated with each saddle point, including the classical vacuum and the various instanton solutions. This amounts to integration over the full set of field fluctuations around each saddle point, with each field integrated over its full range, but possibly deformed into the complex plane. The second step is to express the actual integration contour, along which the path integral over the fields is to be performed, as a (weighted) union of the Lefschetz thimbles for different saddle points [53-55]. This associates a multiplier factor $\zeta$ to each instanton, with which we need to multiply the steepest descent contribution, before we add the contribution to the amplitude. This can sometime be non-trivial, e.g. in the analysis of [31] in two dimensional bosonic string theory, the multiplier factor associated with the D-instanton turned out to be $1 / 2$. Our analysis in sction 4 can be interpreted as part of the first step of the analysis since we integrate all the modes from $-\infty$ to $\infty$ without worrying about whether the actual integration contour involves the whole range. In this section we shall carry out the second step.

The D-instantons are complex solutions in the Euclidean type IIB string theory since the RR scalar field is imaginary for the D-instanton solution [2]. This may lead one to wonder whether the D-instantons contribute to the amplitude at all, since usually the integration contour in the Euclidean field theory runs over real field configurations, and

\footnotetext{
${ }^{8}$ This dependence on $g_{s}$ was first observed in [39].
} 
therefore would seem to miss the D-instanton configurations altogether. However we shall now argue that this is not the correct way to view the D-instantons since they are not regular solutions of supergravity. Instead one should regard the D-instantons as regular solutions in the open string field theory on an unstable D-brane system whose vacuum describes the regular perturbative vacuum [56]. For example a D-instanton in type IIB string theory can be regarded as a kink solution on a non-BPS Euclidean D0-brane or a vortex solution in the euclidean D1-D̄ brane system. These are regular real solutions of the open string field theory and the reason that the solution appears to be complex in the closed string theory is due to the fact that in the Euclidean theory there is a complex contribution to the action of closed and open strings. For example in the non-BPS D0brane action there is a term proportional to $\int \chi d T$, where $\chi$ is the RR scalar and $T$ is the open string tachyon, and in the Euclidean theory this gets a factor of $i$ due to the $d T$ term acquiring an $i$ from the Wick rotated time direction. Since from the open string perspective the D-instantons are real solutions, we conclude that the integration contour over the open string fields include the full steepest descent contour of the D-instanton. Therefore the multiplier factor $\zeta$ is 1 .

\section{4-graviton amplitude}

We shall now compute the leading D-instanton contribution to the four graviton amplitude. Naively, the leading contribution comes from the product of four disk one point functions, with a graviton vertex operator inserted at the center of each disk. However the contribution from such configurations to the four graviton amplitude vanishes due to the left over integration over the $\widetilde{\chi}_{\alpha}$ 's in (4.50). The remedy is to consider a different amplitude where, besides the four graviton vertex operators inserted at the centers of the four disks, we also have $16 \widetilde{\chi}_{\alpha}$ 's as external states [2]. As will be explained below, this gives a contribution to the effective action containing product of $16 \widetilde{\chi}_{\alpha}$ 's and can give a non-zero result after integration over the $\tilde{\chi}_{\alpha}$ 's.

We shall now proceed as follows:

1. We shall first show that the disk amplitude with a single graviton and $n \tilde{\chi}_{\alpha}$ 's vanish for $n=0,2$, so we need at least four $\tilde{\chi}_{\alpha}$ insertions on the disk to get a non-vanishing result. Therefore the $16 \widetilde{\chi}_{\alpha}$ 's must be equally distributed among the four disks.

2. Let $\mathcal{A}_{\alpha \beta \gamma \delta}(e, k) e^{i k . \widetilde{\xi}}$ be the disk amplitude of a single graviton of polarization $e_{\mu \nu}$ and external open string modes $\widetilde{\chi}_{\alpha}, \widetilde{\chi}_{\beta}, \widetilde{\chi}_{\gamma}$ and $\tilde{\chi}_{\delta}$. Note that we have included the dependence of the amplitude on the position $\tilde{\xi}$ of the instanton. This can be summarized by saying that the effective action of the open closed string field theory, after integrating out the $L_{0}>0$ modes, has a term ${ }^{9}$

$$
\frac{1}{4 !} \int \frac{d^{10} k}{(2 \pi)^{10}} e^{i k . \widetilde{\xi}} \mathcal{A}_{\alpha \beta \gamma \delta}(h(k), k) \tilde{\chi}_{\alpha} \tilde{\chi}_{\beta} \widetilde{\chi}_{\gamma} \widetilde{\chi}_{\delta}
$$

\footnotetext{
${ }^{9}$ For writing the effective action (6.1) we need an off-shell continuation of the function $\mathcal{A}_{\alpha \beta \gamma \delta}$. Any off-shell continuation will serve our purpose since eventually we shall evaluate this for on-shell external gravitons.
} 
Eq. (4.1) and (4.50) now show that, after integrating out the open string modes, the closed string effective field theory will have a term

$$
\mathcal{N}_{0} e^{-2 \pi / g_{s}} \int \prod_{\mu} d \widetilde{\xi}^{\mu} \prod_{\alpha=1}^{16} d \widetilde{\chi}_{\alpha} \exp \left[\frac{1}{4 !} \int \frac{d^{10} k}{(2 \pi)^{10}} e^{i k . \widetilde{\xi}} \mathcal{A}_{\alpha \beta \gamma \delta}(h(k), k) \widetilde{\chi}_{\alpha} \widetilde{\chi}_{\beta} \widetilde{\chi}_{\gamma} \widetilde{\chi}_{\delta}\right] .
$$

After expanding the exponential and using the result

$$
\int \prod_{\alpha=1}^{16} d \widetilde{\chi}_{\alpha} \widetilde{\chi}_{\alpha_{1}} \cdots \widetilde{\chi}_{\alpha_{16}}=\epsilon_{\alpha_{1} \cdots \alpha_{16}}, \quad \int \prod_{\mu} d \widetilde{\xi}^{\mu} e^{i \widetilde{\xi} \cdot \sum_{i} k_{i}}=(2 \pi)^{10} \delta^{(10)}\left(\sum_{i} k_{i}\right),
$$

we get a four graviton interaction term in the closed string effective field theory:

$$
\begin{aligned}
\mathcal{N}_{0} e^{-2 \pi / g_{s}} \frac{1}{4 !} \frac{1}{(4 !)^{4}} \epsilon_{\alpha_{1} \beta_{1} \gamma_{1} \delta_{1} \cdots \alpha_{4} \beta_{4} \gamma_{4} \delta_{4}} & \\
& \times \int \frac{d^{10} k_{1}}{(2 \pi)^{10}} \cdots \frac{d^{10} k_{4}}{(2 \pi)^{10}}(2 \pi)^{10} \delta^{(10)}\left(k_{1}+k_{2}+k_{3}+k_{4}\right) \\
& \times \prod_{i=1}^{4} \mathcal{A}_{\alpha_{i} \beta_{i} \gamma_{i} \delta_{i}}\left(h\left(k_{i}\right), k_{i}\right) .
\end{aligned}
$$

This generates the following contribution to the four graviton amplitude with polarizations $e_{\mu \nu}^{(i)}$ and momentum $k_{i}$ with $1 \leq i \leq 4$ :

$$
\begin{aligned}
\mathcal{N}_{0} e^{-2 \pi / g_{s}} \frac{1}{(4 !)^{4}} & \epsilon_{\alpha_{1} \beta_{1} \gamma_{1} \delta_{1} \cdots \alpha_{4} \beta_{4} \gamma_{4} \delta_{4}} \\
& \times \prod_{i=1}^{4} \mathcal{A}_{\alpha_{i} \beta_{i} \gamma_{i} \delta_{i}}\left(e^{(i)}, k_{i}\right)(2 \pi)^{10} \delta^{(10)}\left(k_{1}+k_{2}+k_{3}+k_{4}\right) .
\end{aligned}
$$

Therefore our main task will be to compute $\mathcal{A}_{\alpha \beta \gamma \delta}(e, k)$.

We shall begin by showing that the disk amplitude of a single graviton with polarization $e_{\mu \nu}$ vanishes. The vertex operator in the $(-1,-1)$ picture up to a sign is $2 e_{\mu \nu} c \bar{c} e^{-\phi} e^{-\bar{\phi}} \psi^{\mu} \bar{\psi}^{\nu}$. We place the vertex operator at the point $i$ in the upper half plane and, using the doubling trick, replace $\bar{\psi}^{\nu}(i)$ by $\psi^{\nu}(-i)$. The $\psi$ correlator now produces a factor of $\eta^{\mu \nu}$ which shows that the amplitude is proportional to $\eta^{\mu \nu} e_{\mu \nu}$. This vanishes since the polarization tensor is traceless.

Next we compute the disk amplitude for one graviton and a pair of fermion zero modes $\tilde{\chi}_{\alpha}$ and $\tilde{\chi}_{\beta}$. We insert the graviton vertex operator at $i$ on the upper half plane as before, but convert this to $(0,-1)$ picture by taking the product with the PCO $\mathcal{X}$, represent $\chi_{\alpha}$ by the unintegrated $-1 / 2$ picture vertex operator $c e^{-\phi / 2} S_{\alpha}$ inserted at the origin of the upper half plane, and represent $\chi_{\beta}$ by an integrated $-1 / 2$ picture vertex operator $e^{-\phi / 2} S_{\beta}(z)$ and integrate $z$ along the real axis. The amplitude is proportional to:

$$
\int d z\left\langle 2 e_{\mu \nu} c \bar{c}\left\{\partial X^{\mu}+i k_{\rho} \psi^{\rho} \psi^{\mu}\right\} e^{i k . X} e^{-\bar{\phi}} \bar{\psi}^{\nu}(i) c e^{-\phi / 2} S_{\alpha}(0) e^{-\phi / 2} S_{\beta}(z)\right\rangle_{\mathrm{UHP}} .
$$


We can now use the doubling trick to convert this to a correlation function on the full complex plane:

$$
\int d z\left\langle 2 e_{\mu \nu} c\left\{\partial X^{\mu}+i k_{\rho} \psi^{\rho} \psi^{\mu}\right\} e^{i k . X}(i) c e^{-i k . X} e^{-\phi} \psi^{\nu}(-i) c e^{-\phi / 2} S_{\alpha}(0) e^{-\phi / 2} S_{\beta}(z)\right\rangle_{\text {plane }},
$$

where all the fields are regarded as holomorphic. The $z$ integral may be taken to pass either above or below the origin where $c e^{-\phi / 2} S_{\alpha}$ is inserted since, according to (2.13), the difference between these two choices of contour is proportional to $\gamma_{\alpha \beta}^{\mu}$ which is symmetric under the exchange of $\alpha$ and $\beta$. Since eventually we need to contract this amplitude with $\epsilon_{\alpha \beta \ldots}$, the contribution of this term will vanish. Let us take the contour to pass above the origin. We can now deform this to pick up the residue from the $\psi^{\rho} \psi^{\mu}$ insertion at $i$. Using (2.13) we see that the resulting contribution will be proportional to

$$
\begin{aligned}
& \frac{1}{2} k_{\rho} e_{\mu \nu}\left(\gamma^{\rho \mu}\right)_{\beta}^{\gamma}\left\langle c e^{-\phi / 2} S_{\gamma} e^{i k . X}(i) c e^{-i k \cdot X} e^{-\phi} \psi^{\nu}(-i) c e^{-\phi / 2} S_{\alpha}(0)\right\rangle_{\text {plane }} \\
& \propto k_{\rho} e_{\mu \nu}\left(\gamma^{\rho \mu}\right)_{\beta}^{\gamma}\left(\gamma^{\nu}\right)_{\gamma \alpha},
\end{aligned}
$$

where $\gamma^{\mu_{1} \cdots \mu_{n}}$ is the totally antisymmetric product of $\gamma^{\mu_{1}}, \cdots, \gamma^{\mu_{n}}$, normalized so that it is given by $\gamma^{\mu_{1}} \cdots \gamma^{\mu_{n}}$ when all the $\mu_{i}$ 's are different. After expressing $\gamma^{\rho \mu} \gamma^{\nu}$ as a linear combination of $\gamma^{\mu \nu \rho}, \eta^{\rho \nu} \gamma^{\mu}$ and $\eta^{\mu \nu} \gamma^{\rho}$, we see that (6.8) vanishes using the symmetry and tracelessness of $e_{\mu \nu}$ and the condition $k^{\mu} e_{\mu \nu}=0$.

We shall now compute the amplitude $\mathcal{A}_{\alpha \beta \gamma \delta}(e, k)$ with one canonically normalized graviton and four $\tilde{\chi}$ 's inserted on the disk. During this computation we shall not be careful about factors of $i$ and minus signs since according to (6.5) the result will be raised to fourth power. We shall convert the graviton vertex operator at $i$ to an unintegrated zero picture vertex operator given in (3.12) and call this $V_{C}$ :

$$
V_{C}=2 e_{\mu \nu} \bar{c} c\left\{\partial X^{\mu}+i k_{\rho} \psi^{\rho} \psi^{\mu}\right\}\left\{\bar{\partial} X^{\nu}+i k_{\sigma} \bar{\psi}^{\sigma} \bar{\psi}^{\nu}\right\} e^{i k . X}+\cdots
$$

The $\cdots$ terms have non-zero $\phi$ charge and will not contribute to the correlation function. We denote the unintegrated $-1 / 2$ picture vertex operator of $\tilde{\chi}_{\alpha}$ by $c W_{\alpha}$ where,

$$
W_{\alpha}=e^{-\phi / 2} S_{\alpha} .
$$

The corresponding integrated vertex operator is $W_{\alpha}$. We take the vertex operator of $\tilde{\chi}_{\alpha}$ to be unintegrated, placed at the origin, and those of $\tilde{\chi}_{\beta}, \tilde{\chi}_{\gamma}$ and $\tilde{\chi}_{\delta}$ to be integrated along the real axis. Therefore, according to (3.28), the amplitude will be given by: ${ }^{10}$

$$
\mathcal{A}_{\alpha \beta \gamma \delta}(e, k)=\kappa \pi \mathcal{T} \int d y_{1} d y_{2} d y_{3}\left\langle V_{C}(i) c W_{\alpha}(0) W_{\beta}\left(y_{1}\right) W_{\gamma}\left(y_{2}\right) W_{\delta}\left(y_{3}\right)\right\rangle_{\mathrm{UHP}} .
$$

\footnotetext{
${ }^{10}$ String field theory fixes the assignment of PCOs near each degeneration. This translates to the following simple rule for the amplitude under consideration. If the net number of fermionic open string states that approach each other is even, then their picture number must add up to -1 , while if this is number is odd, then their picture number should add up to $-3 / 2$. We can see that the picture number assignment we have taken is consistent with this rule when two or three open strings come together, but when all four open strings come together, we need to move one of the PCOs from the closed string vertex operator to near the open string vertex operators. The effect of this movement can be computed using the trick of vertical integration following [57], and can be shown to vanish in this case. Therefore (6.11) gives the correct expression for the amplitude.
} 
The factor of $\kappa$ comes from having to express $\psi_{c}$ as $\kappa \phi_{c}$, since the external graviton is taken to be the canonically normalized field. Since $\tilde{\chi}_{\alpha}$ 's appear in the expansion of $\left|\psi_{R}\right\rangle$, it follows from (3.28) that we do not get any extra factor of $g_{s}$.

We can represent the vertex operator $V_{C}$ as

$$
V_{C}(z)=2 e_{\mu \nu} \bar{c}(\bar{z}) c(z) U^{\mu}(z) \bar{U}^{\nu}(\bar{z}),
$$

where $U_{\mu}$ is a holomorphic operator,

$$
U^{\mu}=\left(\partial X^{\mu}+i k_{\rho} \psi^{\rho} \psi^{\mu}\right) e^{i k . X} .
$$

This allows us to use the doubling trick and express the amplitude in terms of correlation functions of holomorphic fields on the full complex plane:

$$
\mathcal{A}_{\alpha \beta \gamma \delta}(e, k)=2 \pi \kappa \mathcal{T} e_{\mu \nu} \int d y_{1} d y_{2} d y_{3}\left\langle c U^{\mu}(i) c U^{\nu}(-i) c W_{\alpha}(0) W_{\beta}\left(y_{1}\right) W_{\gamma}\left(y_{2}\right) W_{\delta}\left(y_{3}\right)\right\rangle,
$$

where it will be understood that due to Dirichlet boundary condition on $X^{\mu}$, the $e^{i k . X}$ factor is replaced by $e^{-i k . X}$ in the expression for $U^{\mu}(-i)$. Due to the symmetry arguments described earlier, the relative positions of the integration contours does not matter. We shall choose the $y_{1}$ contour to be above the real axis and $y_{2}$ and $y_{3}$ contours to be below the real axis with $\operatorname{Im}\left(y_{2}\right)>\operatorname{Im}\left(y_{3}\right)$. The holomorphic correlation functions will be normalized following the open string prescription $(2.21)$, with the $(2 \pi)^{p+1} \delta^{(p+1)}(k)$ factor absent for D-instantons.

We can now deform the $y_{1}$ contour into the upper half plane and the $y_{3}$ contour into the lower half plane to pick residues at $i$ and $-i$ respectively. For this we use the operator product expansion derived from (2.13):

$$
W_{\alpha}(y) U^{\mu}(z)=\frac{1}{y-z} \frac{i}{4} k_{\rho}\left(\gamma^{\rho \mu}\right)_{\alpha}^{\beta} W_{\beta}(z) e^{i k \cdot X}(z) .
$$

Similarly we close the $y_{3}$ contour in the lower half plane, picking up the residue at $-i$. This gives, after including the $(2 \pi)^{2}$ factor from the residue theorem,

$$
\begin{aligned}
& \mathcal{A}_{\alpha \beta \gamma \delta}(e, k)=8 \pi^{3} \kappa \mathcal{T} e_{\mu \nu} \int d y_{2}\left\langle c(i) \frac{i}{4} k_{\rho}\left(\gamma^{\rho \mu}\right)_{\beta}^{\beta^{\prime}} W_{\beta^{\prime}}(i) e^{i k . X}(i)\right. \\
&\left.c(-i) \frac{i}{4} k_{\sigma}\left(\gamma^{\sigma \nu}\right)_{\delta}^{\delta^{\prime}} W_{\delta^{\prime}}(-i) e^{-i k \cdot X}(-i) c(0) W_{\alpha}(0) W_{\gamma}\left(y_{2}\right)\right\rangle .
\end{aligned}
$$

Next we can deform the $y_{2}$ contour to pick the residue at $-i$ using the operator product expansion derived from (2.13):

$$
W_{\gamma}(y) W_{\delta^{\prime}}(z)=\frac{1}{y-z} i\left(\gamma^{\tau}\right)_{\gamma \delta^{\prime}} e^{-\phi} \psi_{\tau}(z) .
$$

This gives

$$
\begin{aligned}
& \mathcal{A}_{\alpha \beta \gamma \delta}(e, k)=16 \pi^{4} \kappa \mathcal{T} e_{\mu \nu}\left\langle c(i) \frac{i}{4} k_{\rho}\left(\gamma^{\rho \mu}\right)_{\beta}^{\beta^{\prime}} W_{\beta^{\prime}}(i) e^{i k . X}(i)\right. \\
&\left.c(-i) \frac{i}{4} k_{\sigma}\left(\gamma^{\sigma \nu} \gamma^{\tau}\right)_{\delta \gamma} e^{-\phi} \psi_{\tau}(-i) e^{-i k . X}(-i) c(0) W_{\alpha}(0)\right\rangle .
\end{aligned}
$$


Finally we can use the result

$$
\left\langle c(i) W_{\beta^{\prime}}(i) e^{i k \cdot X}(i) c(0) W_{\alpha}(0) c(-i) e^{-\phi} \psi_{\tau}(-i) e^{-i k \cdot X}(-i)\right\rangle=\frac{i}{2}\left(\gamma_{\tau}\right)_{\beta^{\prime} \alpha},
$$

and drop all factors of $i$ since we have not kept track of these factors even in the interaction vertex (3.28) that we have been using. This gives,

$$
\mathcal{A}_{\alpha \beta \gamma \delta}(e, k)=\frac{1}{2} \pi^{4} \kappa \mathcal{T} e_{\mu \nu}\left(\gamma^{\rho \mu} \gamma_{\tau}\right)_{\beta \alpha}\left(\gamma^{\sigma \nu} \gamma^{\tau}\right)_{\delta \gamma} k_{\rho} k_{\sigma}
$$

Using the result,

$$
\gamma^{\rho \mu} \gamma^{\tau}=\gamma^{\rho \mu \tau}+\eta^{\mu \tau} \gamma^{\rho}-\eta^{\rho \tau} \gamma^{\mu},
$$

and the fact that $\gamma^{\rho}, \gamma^{\mu}$ are symmetric matrices and that we eventually anti-symmetrize the amplitude under the permutation of $\alpha, \beta, \gamma, \delta$, we can write

$$
\mathcal{A}_{\alpha \beta \gamma \delta}(e, k)=\frac{1}{2} \pi^{4} \kappa \mathcal{T} e_{\mu \nu}\left(\gamma_{\tau}^{\rho \mu}\right)_{\beta \alpha}\left(\gamma^{\sigma \nu \tau}\right)_{\delta \gamma} k_{\rho} k_{\sigma} .
$$

Using (6.5) and (6.22) we now get the single D-instanton contribution to the 4-graviton amplitude:

$$
\mathcal{N}_{0} e^{-2 \pi / g_{s}}\left(\frac{1}{2} \pi^{4} \kappa \mathcal{T}\right)^{4} \frac{1}{(4 !)^{4}} \epsilon^{\alpha_{1} \beta_{1} \gamma_{1} \delta_{1} \cdots \alpha_{4} \beta_{4} \gamma_{4} \delta_{4}} \prod_{i=1}^{4}\left\{e_{\mu_{i} \nu_{i}}^{(i)}\left(\gamma^{\rho_{i} \mu_{i}}{ }_{\tau_{i}}\right)_{\beta_{i} \alpha_{i}}\left(\gamma^{\sigma_{i} \nu_{i} \tau_{i}}\right)_{\delta_{i} \gamma_{i}} k_{\rho_{i}}^{(i)} k_{\sigma_{i}}^{(i)}\right\}
$$

We now use the result: ${ }^{11}$

$$
\epsilon^{\alpha_{1} \beta_{1} \gamma_{1} \delta_{1} \cdots \alpha_{4} \beta_{4} \gamma_{4} \delta_{4}} \prod_{i=1}^{4}\left\{e_{\mu_{i} \nu_{i}}^{(i)}\left(\gamma_{\tau_{i}}^{\rho_{i} \mu_{i}}\right)_{\beta_{i} \alpha_{i}}\left(\gamma^{\sigma_{i} \nu_{i} \tau_{i}}\right)_{\delta_{i} \gamma_{i}} k_{\rho_{i}}^{(i)} k_{\sigma_{i}}^{(i)}\right\}=(4 !)^{4} 2^{12} K_{c}
$$

where

$$
K_{c}\left(e_{1}, e_{2}, e_{3}, e_{4}\right)=t^{\mu_{1} \nu_{1} \cdots \mu_{4} \nu_{4}} t^{\rho_{1} \sigma_{1} \cdots \rho_{4} \sigma_{4}} \prod_{j=1}^{4} e_{\mu_{j} \rho_{j}}^{(j)} k_{\nu_{j}}^{(j)} k_{\sigma_{j}}^{(j)},
$$

and $t^{\rho_{1} \sigma_{1} \cdots \rho_{4} \sigma_{4}}$ is defined via the relation:

$$
\begin{aligned}
t^{\mu_{1} \nu_{1} \cdots \mu_{4} \nu_{4}} \prod_{j=1}^{4} f_{\mu_{j}}^{(j)} k_{\nu_{j}}^{(j)}= & \frac{1}{8}\left[4 \operatorname{Tr}\left(M_{1} M_{2} M_{3} M_{4}\right)-\operatorname{Tr}\left(M_{1} M_{2}\right) \operatorname{Tr}\left(M_{3} M_{4}\right)\right] \\
& +2 \text { permutations } \\
M_{i \mu \nu} \equiv & k_{\mu}^{(i)} f_{\nu}^{(i)}-f_{\mu}^{(i)} k_{\nu}^{(i)}
\end{aligned}
$$

This gives the amplitude to be

$$
\mathcal{N}_{0} e^{-2 \pi / g_{s}} 2^{8}\left(\pi^{4} \kappa \mathcal{T}\right)^{4} K_{c} .
$$

Using (3.31), (3.34) and (4.50), and the result $\zeta=1$, we can express this as:

$$
e^{-2 \pi / g_{s}} i g_{s}^{7 / 2} 2^{-18} \pi^{-26} 2^{8}\left(\pi^{4} \times 2^{3} \pi^{7 / 2} g_{s} \times 2 \pi / g_{s}\right)^{4} K_{c}=i e^{-2 \pi / g_{s}} 2^{6} \pi^{8} g_{s}^{7 / 2} K_{c} .
$$

This reproduces (1.2). We shall check in sction 7 that it agrees with the prediction of S-duality.

\footnotetext{
${ }^{11}$ This result was stated in [2] up to an overall normalization factor. The proportionality between the two sides of (6.24) follows from space-time supersymmetry which fixes the tensor structure of the four graviton amplitude. We have computed the normalization by numerically evaluating both sides for special cases.
} 


\section{Prediction for the D-instanton contribution to the four graviton am- plitude from duality}

We shall now derive the prediction for the same amplitude using S-duality of type IIB string theory. In the convention of [40], which agrees with ours, the tree level scattering amplitude takes the form:

$$
\frac{i}{4} \kappa^{2} K_{c}\left[\frac{64}{s t u}+2 \zeta(3)\right](2 \pi)^{10} \delta^{(10)}\left(k_{1}+k_{2}+k_{3}+k_{4}\right) .
$$

The first term can be identified as the contribution to the scattering amplitude from the Einstein-Hilbert action [58] and can be used to check the overall normalization of (7.1). This is S-duality invariant by itself as can be seen by converting this result to the Einstein frame by multiplying this be a factor of $1 / g_{s}^{2}$. The second term can be identified as the contribution from a new term in the action proportional to the fourth power of the Riemann tensor [32]. This term is not S-duality invariant by itself, but admits a completion to an Sduality invariant action by adding a one loop and non-perturbative terms [2]. This modifies the four graviton amplitude to: ${ }^{12}$

$\frac{i}{4} \kappa^{2} K_{c}\left[\frac{64}{s t u}+2 \zeta(3)+\frac{2 \pi^{2}}{3} g_{s}^{2}+4 \pi g_{s}^{3 / 2}\left\{e^{2 \pi i \tau}+e^{-2 \pi i \bar{\tau}}\right\}+\cdots\right](2 \pi)^{10} \delta^{(10)}\left(k_{1}+k_{2}+k_{3}+k_{4}\right)$,

where,

$$
\tau=a+\frac{i}{g_{s}}
$$

with $a$ being the expectation value of the RR scalar field. The coefficient of $e^{2 \pi i \tau}$ gives the single D-instanton contribution to the amplitude and the coefficient of $e^{-2 \pi i \bar{\tau}}$ gives the anti-D-instanton contribution. Therefore the expected contribution to the amplitude from a single D-instanton is:

$$
e^{2 \pi i a} e^{-2 \pi / g_{s}} \frac{i}{4} \kappa^{2} K_{c} 4 \pi g_{s}^{3 / 2}=i e^{2 \pi i a} e^{-2 \pi / g_{s}} 2^{6} \pi^{8} g_{s}^{7 / 2} K_{c},
$$

where in the last step we have used (3.34). This agrees with (6.28) for vanishing RR scalar.

\section{Generalizations}

In this section we shall discuss possible generalizations of our analysis.

The computation of the D-instanton amplitude in this paper consisted of two parts. The subtle part involved the computation of the normalization constant $\mathcal{N}$ in sction 4 . This part of the computation will be the same for all single D-instanton amplitudes in type IIB string theory, irrespective of the number of external lines, their nature and the order of $g_{s}$ to which we want to compute the amplitude. The second part of the analysis, that in sction 6 , is specific to the amplitude we are interested in, and will have to be redone for a different amplitude.

\footnotetext{
${ }^{12}$ The original paper [2] had a typographical error in the coefficient of the $e^{2 \pi i \tau}$ term, but the correct coefficient can be found in later papers e.g. in [3].
} 
Next we shall discuss generalization of this analysis to other theories. Our analysis in this paper, as well as in [31], simplified since the contribution from the $L_{0}>0$ states in the integrand of the annulus partition function (4.3) cancelled and we had to deal with a finite number of modes. However this is not necessary. Let us suppose that the annulus partition function $A$ has the form:

$$
A=\int_{0}^{\infty} \frac{d t}{2 t} f(t) .
$$

Then the key property that is needed to generalize our analysis is the vanishing of $f(t)$ in the $t \rightarrow 0$ limit so that the integral does not have any divergence from the lower end. This is valid in any string theory without closed string tachyons, since the contribution from the small $t$ region can be interpreted as coming from the infrared region of a single loop of closed strings emitted and absorbed by the D-instanton. This contribution is finite as long as there are no closed string tachyons and we have more than two non-compact dimensions. For this reason we only have to deal with possible divergences from the $t \rightarrow \infty$ region associated with the tachyonic and zero modes of the open string. Let us express the tachyonic and the zero mode contributions to $f(t)$ as

$$
\sum_{i=1}^{m} e^{-2 \pi t h_{i}^{b}}-\sum_{j=1}^{n} e^{-2 \pi t h_{j}^{f}},
$$

where the sum runs over the non-positive $h_{i}^{b}$ and $h_{j}^{f}$ values. If $m$ and $n$ are equal, then (8.2) vanishes at $t \rightarrow 0$, and we can analyze the contribution of (8.2) to $e^{A}$ by representing it as integral over bosonic and fermionic modes as in this paper and deal with the zero modes appropriately. For the tachyonic modes we can simply use the steepest descent contour as in [31]. For the rest of the contribution to $f(t)$, coming from $L_{0}>0$ modes, we can evaluate the integral over $t$ in (8.1) explicitly (if necessary numerically) and get a finite result since the integrand vanishes sufficiently fast both as $t \rightarrow 0$ and $t \rightarrow \infty$. If on the other hand $m$ and $n$ in (8.2) are not equal, we can simply include the contribution from appropriate number of positive $h_{i}^{b}$ or $h_{j}^{f}$ modes in the sum in (8.2) to make them equal, and then proceed as before. One can easily verify that the final result is independent of which set of positive $h_{i}$ values we include in the sum in (8.2).

This gives a systematic procedure for computing the contribution of a D-instanton to an amplitude from the steepest descent contour (Lefschetz thimble) of the instanton. However we also need to understand how the steepest descent contour fits inside the full integration contour. If the instanton is a real solution in open string field theory on unstable D-brane system and has no tachyons, then we expect the full steepest descent contour to be part of the integration contour and the multiplier factor will be unity. Otherwise we need to do further analysis to evaluate the multiplier factor.

This shows that the ability to carry out systematic computation of D-instanton correction to string theory amplitudes does not rely on supersymmetry but on the ultraviolet finiteness of string theory. Finally we would like to note that the arguments given above hold also for other Euclidean D-branes as long as they are wrapped on compact cycles and have more than two transverse non-compact directions. Therefore the same method could be used to compute the contribution to the superpotential induced by Euclidean D-branes in $\mathrm{N}=1$ supersymmetric string compactification. 


\section{Acknowledgments}

I wish to thank Bruno Balthazar, Anirban Basu, Rajesh Gopakumar, Michael Green, Sitender Pratap Kashyap, Victor Rodriguez, Jorge Russo, Congkao Wen, Xi Yin and Barton Zwiebach for useful discussions and Bogdan Stefanski for critical comments on an earlier version of this manuscript. This work was supported in part by the Infosys chair professorship and the J. C. Bose fellowship of the Department of Science and Technology, India.

\section{A Sphere four point function from sewing of two three point functions}

In this appendix we shall review how connecting a pair of three point interaction vertices by the propagator (3.15) generates the four point amplitude with the normalization factor given in (3.16). For simplicity we shall illustrate this in the context of bosonic string theory, but the same analysis can be carried out for superstring theory.

Let us suppose that the three point interaction vertices are described by a three point function on the sphere with vertex operators placed at 0,1 and $\infty$. We shall denote by $z$ the global coordinate on the complex plane and choose the local coordinate at 0 to be $z$ and that at $\infty$ to be $-1 / z$. This choice is not symmetric under the permutation of the vertex operators, but will serve to demonstrate the main point of the analysis, i.e. to determine the normalization given in (3.16). We now denote the global coordinates associated with the two three point vertices by $z$ and $z^{\prime}$ and sew the puncture at 0 of the first interaction vertex with the puncture at $\infty$ of the second interaction vertex using the sewing parameter

$$
q=e^{-s+i \theta},
$$

where $s$ and $\theta$ are the parameters introduced in sction 3.1. This gives

$$
z\left(-\frac{1}{z^{\prime}}\right)=q
$$

i.e. $z=-q z^{\prime}$. Therefore in the $z$ plane the punctures at $z^{\prime}=0$ and $z^{\prime}=1$ are located at 0 and $-q$ respectively. The amplitude obtained by sewing two three point functions with the propagator (3.15) is now given by:

$$
i(4 \kappa)^{2}\left(\frac{1}{4 \pi}\right) \int_{0}^{\infty} d s \int_{0}^{2 \pi} d \theta\left\langle\bar{c} c W_{1}(1) \bar{c} c W_{2}(\infty) \oint d z z b(z) \oint d \bar{z} \bar{z} \bar{b}(\bar{z}) \bar{c} c W_{3}(0) \bar{c} c W_{4}(-q)\right\rangle,
$$

where $\oint$ is a contour enclosing the points 0 and $-q$ and $W_{i}$ 's are dimension $(1,1)$ primaries in the matter sector. The $4 \kappa$ factors come from the three point functions as in sction 3.1 and the $i$ is the usual factor in the expression for the S-matrix. We can now carry out the contour integrals to express this as:

$$
\frac{4 i \kappa^{2}}{\pi} \int_{0}^{\infty} d s \int_{0}^{2 \pi} d \theta\left\langle\bar{c} c W_{1}(1) \bar{c} c W_{2}(\infty) \bar{c} c W_{3}(0)|q|^{2} W_{4}(-q)\right\rangle .
$$

Defining $w=-q=-e^{-s+i \theta}$ as the location of the fourth vertex operator and $d^{2} w=2 d x d y$ for $w=x+i y$, we can express this as

$$
\frac{2 i \kappa^{2}}{\pi} \int d^{2} w\left\langle\bar{c} c W_{1}(1) \bar{c} c W_{2}(\infty) \bar{c} c W_{3}(0) W_{4}(w)\right\rangle .
$$

This reproduces (3.16). 


\section{B Comparison of the two definitions of the brane tension}

In this appendix we shall check that the brane tension $\mathcal{T}$ that enters (3.26) agrees with the usual definition based on the low energy effective action. For this we recall that the presence of a $\mathrm{D} p$-brane gives a contribution to the action of the form:

$$
-\mathcal{T} \int d^{p+1} x \sqrt{-\operatorname{det} G} e^{-\Phi}
$$

where $G$ denotes the string metric along the brane and $\Phi$ is the dilaton field. Using (3.9), and assuming that the $\mathrm{D} p$-brane is placed at the origin of the transverse coordinates, (B.1) leads to the following contribution to the action linear in $h_{\mu \nu}$ and $\Phi$ :

$$
-\mathcal{T} \int \frac{d^{9-p} k_{\perp}}{(2 \pi)^{9-p}}\left[\kappa \sum_{\mu, \nu=0}^{p} \eta^{\mu \nu} h_{\mu \nu}\left(0, k_{\perp}\right)-\Phi\left(0, k_{\perp}\right)\right],
$$

where $k_{\perp}$ denotes components of momenta transverse to the brane and we have used the same symbol $\Phi$ to label the Fourier transform of the dilaton field.

We can now compare this with the terms linear in $h_{\mu \nu}$ obtained from (3.26) using the expansion of $\left|\psi_{c}\right\rangle=\kappa\left|\phi_{c}\right\rangle$ from (3.6). Let us denote these coefficients by $h_{\mu \nu}^{\prime}$ instead of the same symbol $h_{\mu \nu}$ that appears in the expansion of the metric. This gives a term in the action of the from:

$$
\frac{1}{2} \kappa \mathcal{T} \int \frac{d^{10} k}{(2 \pi)^{10}} h_{\mu \nu}^{\prime}(k)\left\langle\left(-2 c_{0}^{-} c \bar{c} e^{-\phi} \psi^{\mu} e^{-\bar{\phi}} \bar{\psi}^{\nu} e^{i k . X}(0)\right)\right\rangle_{D},
$$

where

$$
c_{0}^{-}=\frac{1}{2}\left(\oint d w w^{-2} c(w)-\oint d \bar{w} \bar{w}^{-2} \bar{c}(\bar{w})\right),
$$

with the contours evaluated around the origin of the disk and containing factors of $( \pm 2 \pi i)^{-1}$. One can map this into the correlation function on the upper half plane by making appropriate transformation of coordinates and then use the doubling trick and (2.21) to evaluate the matrix element. The result is,

$$
\frac{1}{2} \kappa \mathcal{T} \int \frac{d^{9-p} k_{\perp}}{(2 \pi)^{9-p}}\left[-\sum_{\mu=0}^{p} \eta^{\mu \nu} h_{\mu \nu}^{\prime}\left(0, k_{\perp}\right)+\sum_{\mu=p+1}^{9} \eta^{\mu \nu} h_{\mu \nu}^{\prime}\left(0, k_{\perp}\right)\right],
$$

where the relative minus sign between the two terms in the square bracket is due to the difference in the boundary condition on the $\psi^{\mu}$ 's for $\mu$ tangential and transverse to the D-brane. Similarly (3.26) can be used to calculate the term linear in the scalar $\Psi$ that multiplies the state $\left(\beta_{-1 / 2} \bar{\gamma}_{-1 / 2}+\bar{\beta}_{-1 / 2} \gamma_{-1 / 2}\right) c_{1} \bar{c}_{1}|-1,-1\rangle$ in the expansion of the string field. By choosing the normalization of $\Psi$ appropriately we can express this as:

$$
\frac{1}{2} \kappa \mathcal{T} \int \frac{d^{9-p} k_{\perp}}{(2 \pi)^{9-p}} \Psi(k)
$$

In order to compare (B.2) with the sum of (B.5) and (B.6), we need to know the relation between the fields $\left(h_{\mu \nu}, \Phi\right)$ and $\left(h_{\mu \nu}^{\prime}, \Psi\right)$. Since $h_{\mu \nu}$ and $h_{\mu \nu}^{\prime}$ are known to transform in the 
same way under the linearized gauge transformation laws in supergravity and closed string field theory respectively, they can differ at most by a term proportional to the scalar field $\Phi$. Therefore the general form of the relationship between the two sets of fields takes the form:

$$
h_{\mu \nu}^{\prime}=h_{\mu \nu}+a \Phi \eta_{\mu \nu}, \quad \Psi=b \Phi+c \sum_{\mu=0}^{9} \eta^{\mu \nu} h_{\mu \nu},
$$

for some constants $a, b$ and $c$. Note that for non-zero $c, \Psi$ transforms under gauge transformation - indeed this can be seen directly using the linearized gauge transformation laws of closed string field theory. We can find the constants $a, b$ and $c$ by comparing the action and gauge transformation laws of the low energy supergravity with the action and gauge transformation laws of closed string field theory [59], but we shall take a shortcut. Substituting (B.7) into the sum of (B.5) and (B.6), we get:

$$
\begin{aligned}
-\frac{\kappa \mathcal{T}}{2} \int \frac{d^{9-p} k_{\perp}}{(2 \pi)^{9-p}}\left[(1-c) \sum_{\mu=0}^{p} \eta^{\mu \nu} h_{\mu \nu}\left(0, k_{\perp}\right)-(1+c)\right. & \sum_{\mu=p+1}^{9} \eta^{\mu \nu} h_{\mu \nu}\left(0, k_{\perp}\right) \\
& \left.+\{a(2 p-8)-b\} \Phi\left(0, k_{\perp}\right)\right] .
\end{aligned}
$$

Comparing this with (B.2) for different values of $p$, we see that we must have:

$$
c=-1, \quad a=0, \quad b=2 / \kappa .
$$

We also see that if we had started with some arbitrary normalization on the right hand sides of (B.5) and (B.6), the comparison between (B.8) and (B.2) would have fixed them to be $\kappa \mathcal{T} / 2$ as given in (B.5) and (B.6), This in turn confirms the normalization of (3.26).

Open Access. This article is distributed under the terms of the Creative Commons Attribution License (CC-BY 4.0), which permits any use, distribution and reproduction in any medium, provided the original author(s) and source are credited.

\section{References}

[1] J. Polchinski, Combinatorics of boundaries in string theory, Phys. Rev. D 50 (1994) R6041 [hep-th/9407031] [INSPIRE].

[2] M.B. Green and M. Gutperle, Effects of D instantons, Nucl. Phys. B 498 (1997) 195 [hep-th/9701093] [INSPIRE].

[3] M.B. Green and P. Vanhove, D instantons, strings and M-theory, Phys. Lett. B 408 (1997) 122 [hep-th/9704145] [INSPIRE].

[4] M.B. Green, M. Gutperle and P. Vanhove, One loop in eleven-dimensions, Phys. Lett. B 409 (1997) 177 [hep-th/9706175] [INSPIRE].

[5] E. Kiritsis and B. Pioline, On $R^{4}$ threshold corrections in IIB string theory and $(p, q)$ string instantons, Nucl. Phys. B 508 (1997) 509 [hep-th/9707018] [INSPIRE].

[6] J.G. Russo, An Ansatz for a nonperturbative four graviton amplitude in type IIB superstring theory, Phys. Lett. B 417 (1998) 253 [hep-th/9707241] [InSPIRE]. 
[7] B. Pioline and E. Kiritsis, U duality and D-brane combinatorics, Phys. Lett. B 418 (1998) 61 [hep-th/9710078] [INSPIRE].

[8] J.G. Russo, Construction of $\mathrm{SL}(2, Z)$ invariant amplitudes in type IIB superstring theory, Nucl. Phys. B 535 (1998) 116 [hep-th/9802090] [INSPIRE].

[9] M.B. Green and S. Sethi, Supersymmetry constraints on type IIB supergravity, Phys. Rev. D 59 (1999) 046006 [hep-th/9808061] [INSPIRE].

[10] N.A. Obers and B. Pioline, Eisenstein series and string thresholds, Commun. Math. Phys. 209 (2000) 275 [hep-th/9903113] [INSPIRE].

[11] M.B. Green, H.-h. Kwon and P. Vanhove, Two loops in eleven-dimensions, Phys. Rev. D 61 (2000) 104010 [hep-th/9910055] [INSPIRE].

[12] J.G. Russo, Effects of D-instantons in string amplitudes, Phys. Lett. B 610 (2005) 152 [hep-th/0411035] [INSPIRE].

[13] M.B. Green and P. Vanhove, Duality and higher derivative terms in M-theory, JHEP 01 (2006) 093 [hep-th/0510027] [INSPIRE].

[14] A. Basu, The $D^{* *} 4 R^{4}$ term in type IIB string theory on $T^{2}$ and U-duality, Phys. Rev. D 77 (2008) 106003 [arXiv:0708.2950] [INSPIRE].

[15] A. Basu, The $D^{* *} 6 R^{4}$ term in type IIB string theory on $T^{2}$ and U-duality, Phys. Rev. D 77 (2008) 106004 [arXiv:0712.1252] [inSPIRE].

[16] B. Pioline, $R^{4}$ couplings and automorphic unipotent representations, JHEP 03 (2010) 116 [arXiv: 1001.3647 ] [INSPIRE].

[17] M.B. Green, J.G. Russo and P. Vanhove, Automorphic properties of low energy string amplitudes in various dimensions, Phys. Rev. D 81 (2010) 086008 [arXiv:1001.2535] [INSPIRE].

[18] M.B. Green, S.D. Miller, J.G. Russo and P. Vanhove, Eisenstein series for higher-rank groups and string theory amplitudes, Commun. Num. Theor. Phys. 4 (2010) 551 [arXiv: 1004.0163] [INSPIRE].

[19] M.B. Green, S.D. Miller and P. Vanhove, Small representations, string instantons, and Fourier modes of Eisenstein series, J. Number Theor. 146 (2015) 187 [arXiv:1111.2983] [INSPIRE].

[20] O. Hohm and H. Samtleben, Exceptional form of D=11 supergravity, Phys. Rev. Lett. 111 (2013) 231601 [arXiv:1308.1673] [INSPIRE].

[21] M.B. Green, S.D. Miller and P. Vanhove, $S L(2, \mathbb{Z})$-invariance and D-instanton contributions to the $D^{6} R^{4}$ interaction, Commun. Num. Theor. Phys. 09 (2015) 307 [arXiv:1404.2192] [INSPIRE].

[22] B. Pioline, $D^{6} R^{4}$ amplitudes in various dimensions, JHEP 04 (2015) 057 [arXiv: 1502.03377] [INSPIRE].

[23] Y. Wang and X. Yin, Constraining higher derivative supergravity with scattering amplitudes, Phys. Rev. D 92 (2015) 041701 [arXiv:1502.03810] [INSPIRE].

[24] G. Bossard and A. Kleinschmidt, Loops in exceptional field theory, JHEP 01 (2016) 164 [arXiv: 1510.07859] [INSPIRE].

[25] G. Bossard and A. Kleinschmidt, Cancellation of divergences up to three loops in exceptional field theory, JHEP 03 (2018) 100 [arXiv:1712.02793] [INSPIRE]. 
[26] B. Balthazar, V.A. Rodriguez and X. Yin, ZZ instantons and the non-perturbative dual of $c=1$ string theory, arXiv:1907.07688 [INSPIRE].

[27] B. Balthazar, V.A. Rodriguez and X. Yin, Multi-instanton calculus in $c=1$ string theory, arXiv: 1912.07170 [INSPIRE].

[28] A. Sen, Fixing an ambiguity in two dimensional string theory using string field theory, JHEP 03 (2020) 005 [arXiv: 1908.02782] [INSPIRE].

[29] A. Sen, Divergent $\Longrightarrow$ complex amplitudes in two dimensional string theory, JHEP 02 (2021) 086 [arXiv: 2003.12076] [INSPIRE].

[30] A. Sen, D-instantons, string field theory and two dimensional string theory, JHEP 11 (2021) 061 [arXiv: 2012.11624] [INSPIRE].

[31] A. Sen, Normalization of D-instanton amplitudes, JHEP 11 (2021) 077 [arXiv:2101.08566] [INSPIRE].

[32] D.J. Gross and E. Witten, Superstring modifications of Einstein's l Equations, Nucl. Phys. B 277 (1986) 1 [INSPIRE].

[33] S.M. Chester, M.B. Green, S.S. Pufu, Y. Wang and C. Wen, Modular invariance in superstring theory from $\mathcal{N}=4$ super-Yang-Mills, JHEP 11 (2020) 016 [arXiv:1912.13365] [INSPIRE].

[34] A. Guerrieri, J. Penedones and P. Vieira, Where is string theory in the space of scattering amplitudes?, Phys. Rev. Lett. 127 (2021) 081601 [arXiv:2102.02847] [INSPIRE].

[35] M.B. Green and J.H. Schwarz, Supersymmetrical dual string theory. 3. Loops and renormalization, Nucl. Phys. B 198 (1982) 441 [INSPIRE].

[36] M.B. Green, J.H. Schwarz and L. Brink, $N=4$ Yang-Mills and $N=8$ supergravity as limits of string theories, Nucl. Phys. B 198 (1982) 474 [INSPIRE].

[37] A. Sen, D-instanton perturbation theory, JHEP 08 (2020) 075 [arXiv:2002.04043] [INSPIRE].

[38] H. Erbin, C. Maccaferri, M. Schnabl and J. Vošmera, Classical algebraic structures in string theory effective actions, JHEP 11 (2020) 123 [arXiv: 2006.16270] [INSPIRE].

[39] N. Agmon, B. Balthazar, M. Cho, V. A. Rodriguez and X. Yin, to appear, as described in the talk by Minjae Cho at the Workshop on Matrix Models and String theory, https://www.youtube.com/watch?v=0Nj-T8lgB4E.

[40] J. Polchinski, String theory. Volume 2: superstring theory and beyond, Cambridge Monographs on Mathematical Physics, Cambridge University Press, Cambridge U.K. (2007) [INSPIRE].

[41] C. de Lacroix, H. Erbin, S.P. Kashyap, A. Sen and M. Verma, Closed superstring field theory and its applications, Int. J. Mod. Phys. A 32 (2017) 1730021 [arXiv:1703.06410] [InSPIRE].

[42] D. Friedan, E.J. Martinec and S.H. Shenker, Conformal invariance, supersymmetry and string theory, Nucl. Phys. B 271 (1986) 93 [INSPIRE].

[43] E.P. Verlinde and H.L. Verlinde, Multiloop calculations in covariant superstring theory, Phys. Lett. B 192 (1987) 95 [INSPIRE].

[44] P.A. Grassi, G. Policastro and P. van Nieuwenhuizen, An introduction to the covariant quantization of superstrings, Class. Quant. Grav. 20 (2003) S395 [hep-th/0302147] [INSPIRE]. 
[45] B. Zwiebach, Oriented open-closed string theory revisited, Annals Phys. 267 (1998) 193 [hep-th/9705241] [INSPIRE].

[46] S. Faroogh Moosavian, A. Sen and M. Verma, Superstring field theory with open and closed strings, JHEP 01 (2020) 183 [arXiv:1907.10632] [INSPIRE].

[47] E. Witten, Noncommutative geometry and string field theory, Nucl. Phys. B 268 (1986) 253 [INSPIRE].

[48] E. Witten, Interacting field theory of open superstrings, Nucl. Phys. B 276 (1986) 291 [INSPIRE].

[49] H. Kunitomo and Y. Okawa, Complete action for open superstring field theory, PTEP 2016 (2016) 023B01 [arXiv:1508.00366] [INSPIRE].

[50] A. Sen, Universality of the tachyon potential, JHEP 12 (1999) 027 [hep-th/9911116] [INSPIRE].

[51] A. Sen, Cutkosky rules and unitarity (violation) in D-instanton amplitudes, arXiv:2012.00041 [INSPIRE].

[52] M.R. Gaberdiel and B. Zwiebach, Tensor constructions of open string theories. 1: Foundations, Nucl. Phys. B 505 (1997) 569 [hep-th/9705038] [InSPIRE].

[53] M. Mariño, Lectures on non-perturbative effects in large $N$ gauge theories, matrix models and strings, Fortsch. Phys. 62 (2014) 455 [arXiv: 1206.6272] [INSPIRE].

[54] G.V. Dunne and M. Ünsal, What is QFT? Resurgent trans-series, Lefschetz thimbles, and new exact saddles, PoS (LATTICE2015) 010 [arXiv: 1511.05977] [INSPIRE].

[55] I. Aniceto, G. Basar and R. Schiappa, A primer on resurgent transseries and their asymptotics, Phys. Rept. 809 (2019) 1 [arXiv:1802.10441] [INSPIRE].

[56] A. Sen, Tachyon dynamics in open string theory, Int. J. Mod. Phys. A 20 (2005) 5513 [hep-th/0410103] [INSPIRE].

[57] A. Sen, Off-shell amplitudes in superstring theory, Fortsch. Phys. 63 (2015) 149 [arXiv: 1408.0571] [INSPIRE].

[58] S. Sannan, Gravity as the limit of the Type II superstring theory, Phys. Rev. D 34 (1986) 1749 [INSPIRE].

[59] H. Yang and B. Zwiebach, A closed string tachyon vacuum?, JHEP 09 (2005) 054 [hep-th/0506077] [INSPIRE]. 